\title{
Evaluación de metales preciosos (Au y Ag) en zonas de oxidación al noroeste de Artemisa (Cuba)
}

\author{
Ramón Guillermo Pérez-Vázquez ${ }^{1 *} \mathbb{D}$; Roniel Martín-Lago² ${ }^{\mathbb{C}}$
}

Forma de citar: Pérez-Vázquez, R.G.; Martín-Lago, R. (2021). Evaluación de metales preciosos (Au y Ag) en zonas de oxidación al noroeste de Artemisa (Cuba). Boletín de Geología, 43(1), 127-146. https://doi.org/10.18273/revbol.v43n1-2021006

\section{Resumen}

La determinación de contenidos de metales preciosos ( $\mathrm{Au}$ y Ag) en las zonas de oxidación, alteración hidrotermal y cizallamiento presentes en la zona metalogénica Bahía Honda, al noroeste de Artemisa, fue el objetivo primordial de la investigación. La mayoría de estas zonas se localizan sobre y en los alrededores de los yacimientos y/o prospectos cupro - sulfurosos que se extienden desde el macizo de Cajálbana, hacia el oeste, hasta Buena Vista, hacia el este.

El motivo fundamental para emprender la tarea ha sido el creciente interés que ha habido en Cuba a partir del descubrimiento de yacimientos de oro en zonas de oxidación (gossan) de los depósitos de sulfuros tipo sedex, VMS y epitermales, entre los que se encuentran Oro Castellanos, Cobre Mantua, Loma de Hierro y Golden Hill, además de que la inmensa mayoría de los depósitos de cobre y metales base de Cuba son portadores de Au y Ag. La metodología se basó en la realización de itinerarios geológicos, el muestreo y la documentación de los principales afloramientos y muestras de testigos de pozos, además del análisis y la reinterpretación de la información geofísica y geoquímica de los trabajos anteriores. Las muestras fueron analizadas en el laboratorio "José Isaac del Corral" (LACEMI), determinándose mediante análisis combinado de docimasia con espectrometría de absorción atómica los contenidos de Au y Ag. Se realizó también análisis químico por espectrometría de masas con plasma inductivamente acoplado (ICP - MS) a un grupo de elementos acompañantes.

Como resultado de estos trabajos se pudo determinar la presencia de valores significativos de Au en todas las zonas evaluadas, oscilando entre $0,05-2,24 \mathrm{~g} / \mathrm{t}$, lo cual permitió realizar el cálculo de los recursos hipotéticos. Los valores de la concentración de Ag fueron muy bajos en todas las zonas estudiadas.

Palabras clave: Evaluación; Prospección; Oro; Plata; Gossan; Cuba.

\section{Evaluation of precious metals (Au and Ag) at the oxidation zone northwest of Artemisa (Cuba)}

\begin{abstract}
Determination of specific precious metal ( $\mathrm{Au}$ and $\mathrm{Ag}$ ) contents in the oxidation, hydrothermal alteration and shear zones present at the Bahía Honda metallogenic zone, northwest of Artemisa, was the primary aim of the research. Most of these areas are located on and around the deposits and/or cupro - sulphurous target that extend from the Cajálbana massif to the west to Buena Vista to the east.

The main reason for undertaking this task has been the growing interest it has had in Cuba, based on discoveries and assimilation of gold deposits in oxidation zones (gossan), sedex, VMS and epithermal sulfide deposits, among which there are Oro Castellanos, Cobre Mantua, Loma de Hierro and Golden Hill in addition to the vast majority of Cuba's copper and base metal deposits, they are carriers of Au and Ag.

Methodology was based on carrying out geological itineraries, sampling and documentation of the main outcrops and samples obtained during drilling, in addition to the analysis and reinterpretation of geophysical and geochemical information from previous work carried out in the area. The samples were analyzed in the laboratory "José Isaac del Corral" (LACEMI), determining by means of combined analysis of docimasy with atomic absorption spectrometry the contents of $\mathrm{Au}$ and $\mathrm{Ag}$. Chemical analysis was also performed by inductively coupled plasma with plasma inductively coupled (ICP - MS) to a group of accompanying elements.

As a result of these works, it was possible to determine the presence of significant values of Au in all the prospects evaluated, ranging between $0.05-2.24 \mathrm{~g} / \mathrm{t}$, which allowed the calculation of hypothetical resources. Silver concentration values in all studied area were very low.
\end{abstract}

Keywords: Evaluation; Prospecting; Gold; Silver; Gossan; Cuba.

\footnotetext{
${ }^{1}$ Universidad Tecnológica de La Habana “José Antonio Echeverría”, La Habana, Cuba. (*) monicodecuba@gmail.com

${ }^{2}$ Empresa Geominera de Pinar del Río, Cuba. ronielmartin@yahoo.es
} 


\section{Introducción}

La historia de la minería del oro en Cuba ha sido descrita en numerosos trabajos publicados, sin embargo, no ha sido sistematizada acorde a las diferentes etapas del desarrollo socio - económico de la Isla. La producción de oro y su investigación han tenido un gran desarrollo en los últimos años en los cortes de meteorización lateríticos en el mundo, debido a la capacidad técnica del beneficio minero de menas de bajos contenidos y grano fino, así como a la experiencia acumulada en la explotación de los depósitos de tipo gossan, por el método de lixiviación en pila, asociados a yacimientos sulfurosos (Amorós et al., 1981; Freyssinet e Itard, 1997; Díaz-Martínez, 2010; Andreu et al., 2015; Yesares et al., 2015; Pérez-Vázquez et al., 2017; Kim et al., 2018; Kalinin et al., 2018; Ozdemir y Sahinoglu, 2018).

Durante el estadio neo - platafórmico o cobertura en Cuba, representado por las rocas y estructuras formadas desde el Eoceno superior tardío y hasta el Holoceno (IturraldeVinent, 2012), se dieron condiciones favorables para la formación de importantes depósitos minerales de oro asociados al enriquecimiento supergénico ('sombreros de hierro') en depósitos de sulfuros tipo sedex, VMS y epitermales, que suelen ser ricos en $\mathrm{Au} \mathrm{y} \mathrm{Ag,} \mathrm{así} \mathrm{como} \mathrm{a}$ diversos procesos de concentración aluviales y marinos (Díaz-Martínez, 2010; Pérez-Vázquez et al., 2017; Álvarez-Ortiz et al., 2019).

En Cuba ha habido un creciente interés en la exploración de metales preciosos a partir del descubrimiento de yacimientos de oro en zonas de oxidación (gossan), entre los que se encuentran Oro Castellanos (sedex), Cobre Mantua (VMS), Loma de Hierro (gossan) y Golden Hill (epitermal) y depósitos de oro clasificados como orogénicos, formados durante la etapa de subducción y colisión del arco de las Antillas Mayores con el continente de América del Norte (Cazañas-Díaz et al., 2017), así como en los depósitos de tipo placer cuyas rocas madres son del complejo ofiolítico, además de los depósitos de sulfuros masivos (Proenza y Melgarejo, 1998; Vila-Sánchez et al., 2004; Nelson et al., 2011; Emsbo et al., 2016; Pérez-Vázquez et al., 2017; CazañasDíaz et al., 2017). Teniendo en cuenta, además, que la inmensa mayoría de los depósitos de cobre y metales base de Cuba son portadores de oro, en los que el oro se encuentra entrampado en la arsenopirita, y que los sombreros de hierro de muchos yacimientos piríticos con metales base en la parte occidental y central de la isla están enriquecidos en oro (Díaz-Martínez, 2010; Pérez-Vázquez et al., 2017; Cazañas-Díaz et al., 2017), es que surge el motivo fundamental para emprender la tarea de realizar un reconocimiento en la zona metalogénica Bahía Honda para evaluar áreas de interés y para proponer realizar futuras prospecciones.

El objetivo de este trabajo fue la determinación de contenidos de metales preciosos ( $\mathrm{Au} \mathrm{y} \mathrm{Ag)} \mathrm{en} \mathrm{las} \mathrm{zonas}$ de interés presentes en la región: zonas de oxidación, de alteración hidrotermal y de cizallamiento. La mayoría de estas zonas se localizan sobre y en los alrededores de los yacimientos y prospectos cupro - sulfurosos de la región y se extienden desde el macizo de Cajálbana, hacia el oeste, hasta Buena Vista, hacia el este. Este trabajo se llevó a cabo con la intención de identificar los lugares de mayor potencial y realizar la evaluación geólogo económica de los depósitos auro - argentíferos, con el interés de su exploración y explotación futura por parte de la unión de empresas geólogo minera, o bien incrementar el valor objeto para una futura negociación bajo condiciones de asociación mixta.

Por primera vez en el área se ejecutaron trabajos dirigidos a la investigación de metales preciosos, pues anteriormente los trabajos estuvieron enfocados a la mineralización cuprífera. Por consiguiente, los gossan de los depósitos de la región no fueron del interés de las investigaciones previas y no se estudiaron con profundidad.

\section{Marco teórico}

\section{Características geográfico - geológicas de la región}

Para la realización del trabajo se contó con los informes de búsqueda y exploración realizados en el área con anterioridad, así como artículos y publicaciones referentes a la misma (Vologdin y Stepanov, 1967; Kulikov, 1971; Escobar, 1973; Álvarez et al., 1991; Martínez et al., 1991). El área de estudio se encuentra ubicada hacia el este del Terreno Guaniguanico, parte noroeste de la provincia de Artemisa, Cuba Occidental; según la división política administrativa, abarca los municipios de La Palma y Bahía Honda (Figura 1).

El área ocupa aproximadamente $370 \mathrm{~km}^{2}$ donde el relieve que predomina es pre montañoso en su porción septentrional con elevaciones de inclinaciones abruptas, de acceso y movilidad difíciles. Actualmente la economía es mayoritariamente agrícola, destacándose la caña de azúcar, área forestal, café y otros cultivos varios, pero tuvo un desarrollo minero amplio en la década del 40 del pasado siglo (Kulikov, 1971; Vologdin y Stepanov, 1967) debido a la existencia 
de varios yacimientos y prospectos minerales, y posteriormente, a partir de 1979, con la explotación del yacimiento Júcaro, actualmente inactivo (Kulikov, 1971; Estévez-Cruz, 2000). Los principales centros poblacionales del área son La Mulata, Sanguily, Las Cadenas, Las Pozas, Harlem, Orozco y el municipio cabecera de Bahía Honda.

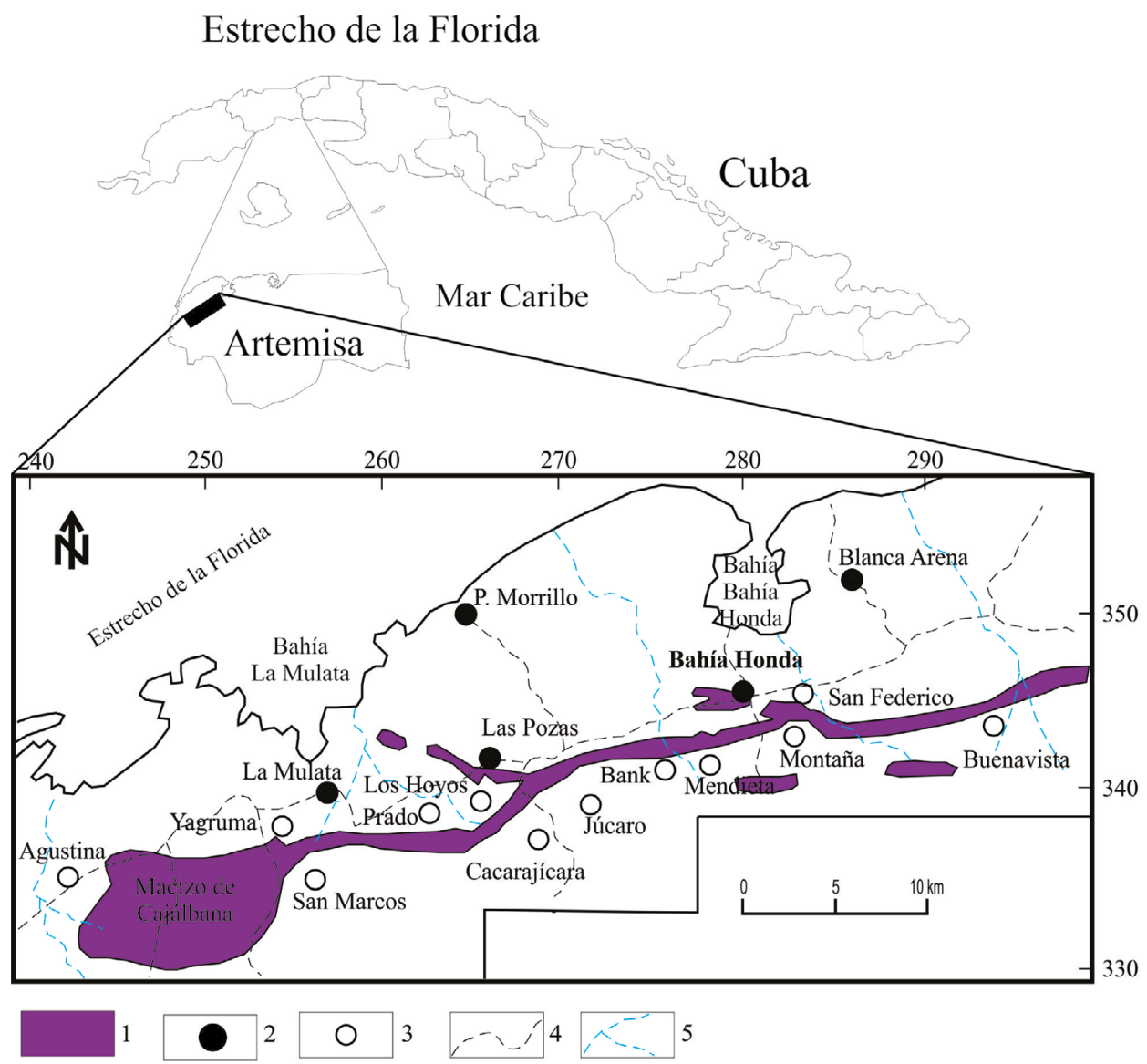

Figura 1. Mapa de ubicación geográfica y de yacimientos del distrito metalogénico Bahía Honda. Escala grafica en km. 1. Complejo ofiolítico. 2. Poblados. 3. Yacimientos y prospectos. 4. Carreteras. 5. Ríos principales.

La región se encuentra dentro de la zona metalogénica Bahía Honda (Simón, 1987) (Figura 1), en la que se localizan depósitos vulcanógenos de sulfuros masivos (VMS tipo Chipre) con mineralización cuprífera sulfurosa con $\mathrm{Zn}$ y $\mathrm{Au}$ - Ag que han sufrido una intensa alteración superficial, en un contexto de magmatismo toleítico (Kulikov, 1971; Cruz-Gámez y SimónMéndez, 1997; Álvarez-Ortiz et al., 2019). Además, esta zona metalogénica se puede separar en dos distritos metalogénicos basados en el criterio formacional (Kulikov, 1971; Simón, 1987; Barrios, 1995; Cazañas-Díaz et al., 2017): (a) Distrito Metalogénico Júcaro - Buena Vista, y $(b)$ Distrito Metalogénico Yagruma. El Distrito Metalogénico Júcaro - Buena Vista se desarrolla en la parte media de la Formación
Encrucijada, de edad $\mathrm{K}_{1}{ }^{\text {al }}-\mathrm{K}_{2}{ }^{\text {ce }}$ (Instituto de Geología y Paleontología, 2013). Son cuerpos estratiformes y de stockwork en lavas basálticas con intercalaciones piroclásticas y se relacionan con procesos de alteración como son: cuarcificación, cloritización y hematización, con una zonación bastante clara. Entre ellos tenemos Júcaro, Cacarajícara, Mendieta - Banks, Buena Vista, Montaña y San Federico. Por otro lado, el Distrito Metalogénico Yagruma se desarrolla en la parte baja de la Formación Encrucijada $\left(\mathrm{K}_{1}^{\text {al }}-\mathrm{K}_{2}{ }^{\mathrm{ce}}\right)$. Las rocas encajantes son, fundamentalmente, diabasas y lavas basálticas, así como paquetes de rocas piroclásticas subordinadas. Los cuerpos de sulfuros en forma de stockwork son los más comunes. Entre ellos tenemos Yagruma, San Marcos y Agustina (Figura 1). 
Geológicamente la región se ha interpretado como un terreno alóctono (Pardo, 1975; Pszczólkowski, 1981; Cobiella-Reguera, 2008), originado en la cuenca de interarco de San Diego de los Baños (Cruz-Gámez y Simón-Méndez, 1997) durante la evolución del arco insular cubano del Cretácico $\left(\mathrm{K}_{1}\right.$ Aptiano $-\mathrm{K}_{2}$ Campaniano). Por otra parte, la zona es considerada como una sub - zona de la Zona Estructuro - Facial Zaza. Esta sub - zona se dispone de manera sublatitudinal en forma de faja de $12-16 \mathrm{~km}$ de ancho por $45 \mathrm{~km}$ de largo, se caracteriza por la presencia de las rocas del complejo ofiolítico, rocas ultrabásicas y basaltos, los cuales, por su firma geoquímica (backarc basin basalt, BABB), son de cuenca de trasarco (Cruz-Gámez y Simón-Méndez, 1997; Proenza y Melgarejo, 1998; Cazañas-Díaz et al., 2017). Además, hay gabros y diabasas, emplazados tectónicamente y de amplio desarrollo e interés metalogénico. La sub-zona además se caracteriza por la yacencia de las secuencias vulcanógenas sedimentarias, representadas por la Formación Encrucijada, $\mathrm{K}_{1}{ }^{\text {al }}-\mathrm{K}_{2}{ }^{\text {ce }}$ (Proenza y Melgarejo, 1998), de alto potencial menífero. En ella es donde se localizan los yacimientos minerales de $\mathrm{Cu}+\mathrm{Zn}(\mathrm{Au}-\mathrm{Ag})$ del tipo Chipre (Figura 2). Esta formación se encuentra tanto al sur como al norte del complejo ofiolítico.
En la región se destaca fisiográficamente la meseta de Cajálbana, con una extensión de $50 \mathrm{~km}^{2}$ y una altitud promedio de $\sim 460 \mathrm{~m}$. Geológicamente se caracteriza por la exposición de rocas ultramáficas serpentinizadas en diferentes grados, así como por procesos de meteorización y lateritización, de composición original peridotítico.

Las particularidades litológico - estratigráficas y tectónicas de la región se encuentran descritas a detalle por Vologdin y Stepanov (1967) y Kulikov (1971). Así, la región se caracteriza por un amplio desarrollo de formaciones del Cretácico, además de las del Paleógeno y del Cuaternario. En la región se han elaborado varias columnas estratigráficas para las secuencias que en ellas aparecen, destacándose las propuestas de Kulikov (1971), Mormil et al. (1980) y Martínez et al. (1991). La edad de las formaciones de la región Bahía Honda va desde la parte inferior del Aptiano hasta el Santoniano (formaciones Encrucijada, Quiñones y Orozco); se trata de rocas de la cuenca marginal del arco volcánico Cretácico. Las formaciones Vía Blanca, Capdevila y Madruga van desde el Cretácico superior hasta el Paleoceno (Figura 2).

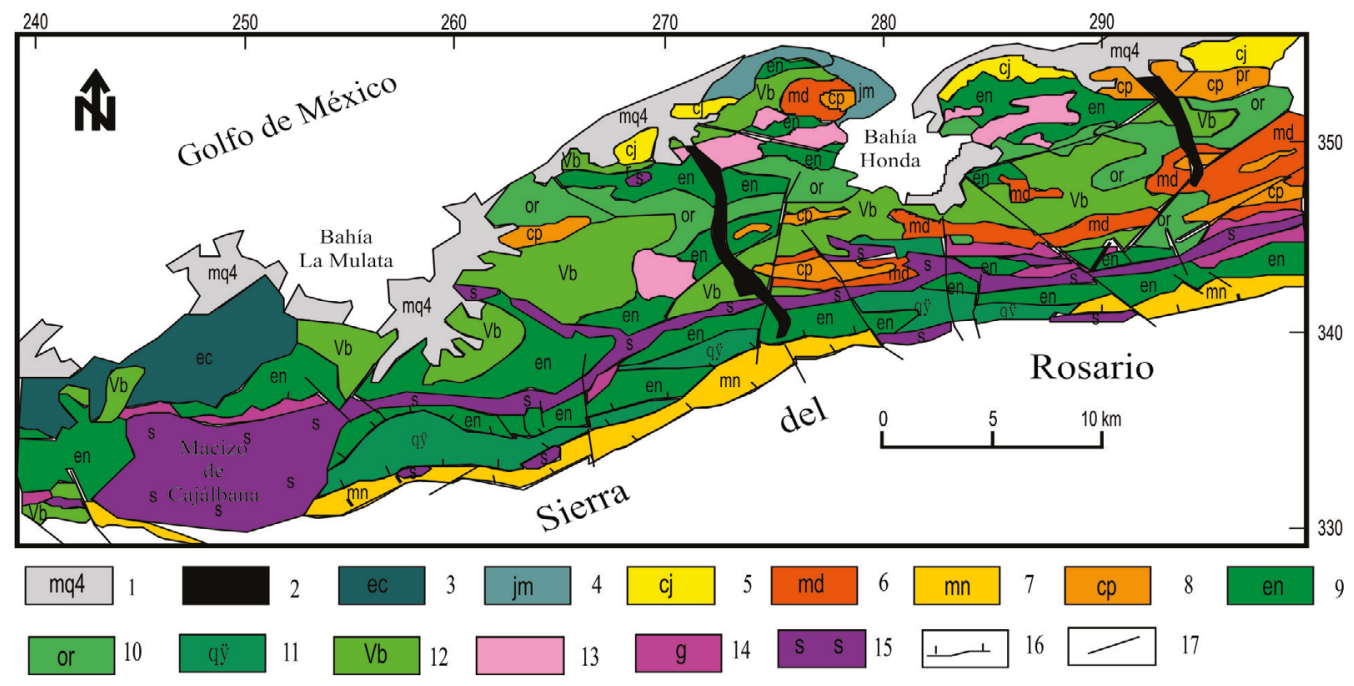
1. Depósitos biogénicos de manglar.
2. Depósitos aluviales
3. Depósitos eluviales
4. Formación Jaimanitas $\mathrm{Q}_{1}{ }^{1}$
5. Formación Cojímar $\mathrm{N}_{1}^{1-2}$
6. Formación Madruga $\mathrm{E}_{1}$
7. Formación Manacas $\mathrm{E}_{2}^{1-2}$
8. Formación Capdevila $\mathrm{P}_{2}{ }^{1}$
9. Formación Encrucijada $\mathrm{K}_{1}^{\mathrm{al}}-\mathrm{K}_{2}^{\mathrm{ce}}$

10. Formación Orozco $\mathrm{K}_{2}^{\text {co-sa }}$

11. Formación Quiñones $\mathrm{K}_{2}^{\text {ce-tu }}$

12. Formación Vía Blanca $\mathrm{K}_{2}^{\text {cp-ma }}$

13. Complejo de diques paralelos

14. Gabros - diabasa del Complejo Ofiolítico

15. Serpentinitas del Complejo Ofiolítico

16. Sobrecorrimientos

17. Fallas

Figura 2. Mapa geológico de la región de Artemisa. Modificado de Pushcharovsky (1988). 
La Formación Encrucijada $\mathrm{K}_{1}{ }^{\mathrm{al}}-\mathrm{K}_{2}$ ce juega un rol muy importante en la geología al encajar los yacimientos y depósitos que se encuentran en la región. Está formada por lavas y brechas basálticas de afinidad toleítico, con pedernal interestratificado, así como calizas micríticas y microorganógenas, en menor cantidad lutitas y limolitas. Está cubierta discordantemente por las formaciones Cojímar $\mathrm{N}_{1}{ }_{1-2}$, Orozco $\mathrm{K}_{2}^{\text {co-sa }}$, Quiñones $\mathrm{K}_{2}^{\text {ce-tu }}$ y Vía Blanca $\mathrm{K}_{2}^{\text {cp-ma }}$.

\section{Antecedentes}

En la región se han efectuado una serie de trabajos encaminados principalmente a la búsqueda de sulfuros, entre ellos citaremos a continuación los más relevantes: en el año 1957 la Atlanta Refining Co. ejecutó un levantamiento aeromagnetométrico a escala 1:200 000, confeccionándose los mapas de isolíneas del campo magnético total y los gradientes horizontal y vertical a escala 1:50 000 (Soloviev et al., 1964). Antes de 1959 la limonita del yacimiento de cobre Yagruma fue explotada por una compañía norteamericana, pero no se conservan datos. Con fines de búsqueda y evaluación se realizaron los trabajos de Vologdin y Stepanov (1967), Kulikov (1971) y Escobar (1973).

La región se encuentra cubierta por un levantamiento geológico a escala 1:50 000 con densidad de 4 a 5 puntos por $\mathrm{km}^{2}$, levantamiento litogeoquímico con análisis espectral de 18 elementos en una red aproximada de $500 \times 500 \mathrm{~m}$, por trabajos geofísicos, fundamentalmente radiometría, magnetometría y gravimetría y por un levantamiento hidrogeológico a la misma escala, así como la realización de trabajos acompañantes a escala 1:10 000 dentro del levantamiento regional (Maximov, 1978). Además, la región está cubierta por un levantamiento aerogeofísico a escala 1:50 000, mediante el cual obtuvo la componente total del campo magnético y los canales de U (Ra), Th y K (Brodoboi et al., 1987). También existen: un mapa gravimétrico a escala 1:100 000, con la base de datos en 1:50 000. Geología y metalogenia de la provincia de Pinar del Río (Mormil y Norman, 1980); y un mapa geológico a escala 1:250 000 de la Academia de Ciencias de Cuba y la URSS (Pushcharovsky, 1988) y el Informe pronóstico de Cobre Júcaro - Buena Vista (Mormil y Norman, 1980).

En resumen, con estas investigaciones podemos afirmar que la composición mineralógica de los yacimientos y prospectos minerales de la región se encuentran bien caracterizadas. En las zonas de oxidación y enriquecimiento secundario se reportan los siguientes minerales (primarios y secundarios): calcosina, covelina, bornita, limonita, azurita, malaquita, cobre nativo, calcopirita, esfalerita, magnetita, hematita, marcasita, siderita, arsenopirita, cubanita, pirrotina, rutilo, cuarzo, clorita, galena y caolinita; mientras que en la mena primaria se encuentran: pirita, calcopirita, esfalerita, cuarzo y clorita (Vologdin y Stepanov, 1967; Kulikov, 1971).

\section{Metodología}

La metodología empleada se seleccionó sobre la base de los objetivos de la investigación y los recursos disponibles para su ejecución. Las principales etapas fueron: (I) elección del modelo geológico conceptual; (II) selección de las áreas para la realización del reconocimiento; (III) cartografía geológica; (IV) perforación; (V) muestreo y análisis de laboratorio.

\section{Elección del modelo geológico conceptual}

Los principales criterios e indicadores de la mineralización primaria (menas sulfurosas), descritos durante los trabajos anteriores, son válidos también para las menas oxidadas (Freyssinet e Itard, 1997; Ozdemir y Sahinoglu, 2018). De esta forma se seleccionaron los siguientes criterios: estratigráficos, geomorfológicos, geofísicos y geoquímicos; estos últimos de mucha importancia ya que en el área se pueden obtener anomalías monoelementales de $\mathrm{Zn}, \mathrm{Cu}$ y $\mathrm{Pb}$. El criterio estratigráfico se pone de manifiesto en el carácter condicionante de la mineralización de tipo sulfurosa estratiforme de la Formación Encrucijada $\mathrm{K}_{1}^{\text {al }}-\mathrm{K}_{2}^{\text {ce }}$. Por su influencia sobre los minerales y rocas del área, se le otorga un interés particular, por cuanto propició el desarrollo de las zonas de oxidación y con ello potenció la acumulación del Au (Taylor y Sylvester, 1982; Freyssinet e Itard, 1997; Andreu et al., 2015; Kalinin et al., 2018; Ozdemir y Sahinoglu, 2018).

Las características geomorfológicas constituyen también un criterio para la localización de la mineralización de $\mathrm{Au}$ yAg en la zona, ya que es muy común encontrarla asociada a las partes positivas del relieve, debido, principalmente, a la estabilidad de la mineralización actual ante los procesos de meteorización y la competencia de las rocas encajantes y la presencia de anomalías geoquímicas y geofísicas reveladas en los trabajos anteriores y actuales. La mineralización se manifiesta, en la mayoría de los casos, como sombreros de hierro vinculados con las cotas mayores, o como depósitos eluvio - deluviales de éstos en las laderas y partes bajas de las elevaciones asociadas con estos cuerpos. 
Las anomalías geofísicas fueron reveladas durante las investigaciones anteriores (Vologdin y Stepanov, 1967; Kulikov, 1971; Brodoboi et al., 1987; Álvarez et al.; 1991; Barrios, 1995). Los principales indicadores de la presencia de zonas de oxidación son las anomalías de PIy magnetométricas. Estas se localizan fundamentalmente en los principales yacimientos y prospectos.

En la región existen claras premisas y datos que constituyen índices de búsqueda, los cuales permitieron a la luz de las nuevas concepciones la realización de este trabajo de reconocimiento.

Los índices empleados fueron:

1. Existencia de depósitos y prospectos de sulfuro masivos con mineralización $\mathrm{Cu}+\mathrm{Zn}(\mathrm{Au}-\mathrm{Ag})$.

2. Presencia sobre estos depósitos de sombreros de hierro o zonas de oxidación con limonitización.

3. Zonas de intenso cizallamiento de las rocas combinado con fallas normales, inversas y de sobrecorrimientos, acompañadas por alteraciones hidrotermales como carbonatización, cloritización, cuarcificación y epidotización.

4. Cartografía de anomalías geoquímicas complejas de $\mathrm{Cu}, \mathrm{Zn}, \mathrm{Pb}, \mathrm{Ag}$, Co, As y geofísicas electromagnéticas, polarización inducida y cuerpo cargado, relacionadas con cuerpos minerales, zonas de fracturación, alteraciones minerales y oxidación de las rocas.

5. Presencia de oro visible rellenando grietas en venas de cuarzo en registros de perforación.

6. Existencia de minas antiguas y activas $y$ escombreras.

Durante los itinerarios geológicos se realizó el muestreo y la documentación de los principales afloramientos y testigos obtenidos durante la perforación. Además, se hizo el análisis y la reinterpretación de la información geofísica y geoquímica de los trabajos anteriores ejecutados en el área. Las muestras fueron analizadas en el laboratorio "José Isaac del Corral", LACEMI, determinándose los contenidos de Au y Ag mediante un análisis combinado de docimasia con espectrometría de absorción atómica. Se realizó también análisis químico por espectrometría de masa con plasma inductivamente acoplado (ICP - MS, por sus siglas en inglés) a un grupo de elementos acompañantes.

\section{Procesamiento e interpretación}

Antes de iniciar la ejecución de los trabajos de campo se realizó una exhaustiva investigación bibliográfica. En este sentido se consultaron los informes geológicos que existían en la Oficina Nacional de Recursos Minerales (ONRM), así como mapas de compilación geoquímicos y geofísicos; además, se interpretaron las fotos aéreas que cubren el área de estudio. Posteriormente pasamos a la determinación de las áreas más interesantes para la realización de las investigaciones de campo. Para la selección de los sectores en los que se ejecutó el reconocimiento, fueron escogidos dos modelos principales de yacimientos minerales, los yacimientos tipo gossan y yacimiento de oro en zona de cizalla, teniendo en cuenta las características geológicas, tectónicas y metalogénicas del área de estudio y la importancia que tienen a nivel mundial las zonas de cizalla para los depósitos auríferos (Fernández de Lara et al., 2001; Zhang, 2020).

En la región de Bahía Honda existe una gran cantidad de depósitos de sulfuros masivos (VMS tipo Chipre) y prospectos minerales, muchos de los cuales forman extensas zonas de oxidación, lo que hace interesante la búsqueda de acumulaciones de metales preciosos en los gossan formados en la zona (Pérez-Vázquez, 2000; Pérez-Vázquez et al., 2017).

En la región (Figura 2), varios autores han teorizado sobre la posibilidad de ocurrencia de metales preciosos en zonas de cizalla (shear), principalmente en las estructuras propias de las zonas de cizalla asociadas al surgimiento de la Falla Pinar y los sistemas de fallas transversales (Cáceres, 1997; Fernández de Lara et al., 2001). Teóricamente, en la zona metalogénica Bahía Honda es posible la ocurrencia de este modelo de yacimiento mineral si se considera la abundancia de zonas de alta deformación, fragmentación y esquistosidad asociadas a dislocaciones de rumbo sublatitudinal. Las rocas en estas zonas generalmente se presentan hidrotermalmente alteradas con silicificación, carbonatización, cloritización y piritización (Kulikov, 1971).

\section{Criterios e índices de búsqueda utilizados para la determinación de las áreas de interés}

Una vez identificadas las principales regularidades que controlan la localización espacial de la mineralización aurífera en la región, se procedió a seleccionar aquellos sectores (Tabla 1) donde convergen los principales criterios e índices geológicos. Sobre esa base se escogió para realizar el reconocimiento en las siguientes áreas (Figuras 3, 4, 5, 6, 7, 8 y 9). 
Tabla 1. Sectores donde se realizó el reconocimiento.

\begin{tabular}{ccccc}
\hline Sectores & \multicolumn{4}{c}{ Coordenadas } \\
\cline { 2 - 5 } & $\mathbf{X}_{\mathbf{1}}$ & $\mathbf{X}_{\mathbf{2}}$ & $\mathbf{Y}_{\mathbf{1}}$ & $\mathbf{Y}_{\mathbf{2}}$ \\
\hline Agustina & 242700 & 244500 & 332300 & 333700 \\
Yagruma & 252200 & 253400 & 337100 & 338000 \\
Prado - Los Hoyos & 262700 & 263900 & 337900 & 338800 \\
Mendieta - Banks & 267700 & 268900 & 337100 & 338500 \\
Montaña & 280100 & 282100 & 342600 & 343800 \\
San Federico & 281000 & 282400 & 341600 & 342500 \\
Buena Vista & 289200 & 290550 & 342200 & 343000 \\
\hline
\end{tabular}

\section{Cartografía geológica}

Se proyectó con el objetivo de precisar y evaluar los diferentes sectores. Éste se realizó a través de los caminos, cañadas y otras vías de acceso a los principales afloramientos para su documentación y muestreo. En el levantamiento se utilizaron las planchetas topográficas a escala 1:10 000 para el amarre de los puntos de documentación y muestreo. A continuación, en la Tabla 2 se aprecia la cantidad de itinerarios geológicos y el número de muestras tomadas.

Tabla 2. Muestras tomadas por sector en los itinerarios.

\begin{tabular}{ccc}
\hline Sector & Itinerarios $\mathbf{( k m )}$ & No. de Muestras \\
\hline Agustina & 6 & 12 \\
Yagruma & 6 & 15 \\
Prado - Los Hoyos & 3 & 14 \\
Mendieta - Banks & 3 & 7 \\
Montaña & - & - \\
San Federico & 3 & 21 \\
Buena Vista & 6 & 39 \\
Otros & 16 & 62 \\
Total & $\mathbf{4 3}$ & $\mathbf{1 7 0}$ \\
\hline
\end{tabular}

Los itinerarios geológicos tuvieron como objetivos revelar la presencia de contenidos significantes de metales preciosos (Au y Ag en menor proporción) a través del reconocimiento geológico y muestreo de todas las zonas de oxidación (limonitización) $\mathrm{y}$ cizallamientos que se presentan sobre y en los alrededores de los yacimientos $\mathrm{y} / \mathrm{o}$ prospectos cupro - sulfurosos de la región, abarcando todas las litologías existentes en estas zonas (rocas del complejo ofiolítico).

\section{Trabajos de perforación}

Se proyectaron $240 \mathrm{~m}$ de perforación distribuidos en 16 pozos. El objetivo de los mismos fue evaluar zonas cubiertas, realizar el reconocimiento en la vertical y determinar la potencia de las zonas de oxidación. Los pozos se realizaron con un ángulo de inclinación de $90^{\circ}$ y un diámetro de $73 \mathrm{~mm}$ lo que garantizó la representatividad de las muestras de los testigos. La profundidad alcanzada por éstos varió entre $6,30 \mathrm{y}$ $15,00 \mathrm{~m}$. El volumen total de perforaciones ejecutadas y el número de muestras de testigo tomada en los distintos sectores se muestra en la Tabla 3.

\section{Métodos de muestreo, tipo de análisis y tipos de muestras}

El muestreo se realizó en afloramientos y en los pozos de perforación. El muestreo de afloramiento se realizó por el método de surco continuo de sección regular, procurándose una profundidad de $3 \mathrm{~cm}$, un ancho de $10 \mathrm{~cm}$ y una longitud de $1 \mathrm{~m}$. El muestreo en los pozos se hizo a testigo completo. A las muestras tomadas se les hizo análisis químico para la determinación de $\mathrm{Au}$ y Ag. Para el Au se utilizó el método combinado 
de ensayo al fuego con Absorción Atómica (AA), mientras que la plata se analizó por el método químico para la determinación de Ag en menas sulfurosas y zonas oxidadas por absorción atómica. A un grupo de las muestras de afloramiento se le realizó análisis (ICP
- MS) de los siguientes elementos: $\mathrm{Sb}, \mathrm{As}, \mathrm{Ga}, \mathrm{Hg}, \mathrm{Bi}$, $\mathrm{Cu}, \mathrm{Zn}, \mathrm{Pb}, \mathrm{Ba}, \mathrm{Fe}$ y $\mathrm{Be}$.

En la Tabla 4 se aprecia el tipo de muestreo realizado y la cantidad de muestras tomadas.

Tabla 3. Volumen de perforación y muestras de testigos.

\begin{tabular}{|c|c|c|c|c|c|}
\hline Sector & Pozos & $\mathbf{X}$ & $\mathbf{Y}$ & Profundidad & Muestras \\
\hline \multirow{3}{*}{ Agustina } & $\mathrm{Pa}-3$ & 242850 & 333240 & 8,80 & 6 \\
\hline & $\mathrm{Pa}-4$ & 243250 & 33250 & 8,40 & 4 \\
\hline & $\mathrm{Pa}-5$ & 243500 & 333040 & 11,60 & 8 \\
\hline \multirow{3}{*}{ Yagruma } & Py-7 & 253160 & 337225 & 6,30 & 6 \\
\hline & Py-8 & 253150 & 337150 & 13,10 & 14 \\
\hline & Py-9 & 252980 & 337390 & 11,10 & 12 \\
\hline \multirow{2}{*}{ Prado - Los Hoyos } & $\mathrm{Pp}-12$ & 263025 & 338315 & 14,00 & 15 \\
\hline & Pp-13 & 263000 & 338400 & 6,30 & 8 \\
\hline \multirow{2}{*}{ Mendieta - Banks } & Pm-19 & 268500 & 337750 & 8,80 & 9 \\
\hline & Pm-20 & 268600 & 337790 & 9,00 & 9 \\
\hline \multirow{2}{*}{ Montaña } & Po-22 & 280555 & 343490 & 11,70 & 14 \\
\hline & Po-27 & 280600 & 343300 & 12,00 & 12 \\
\hline \multirow{2}{*}{ San Federico } & Ps-23 & 281960 & 342310 & 10,00 & 11 \\
\hline & Ps-24 & 282010 & 342250 & 11,30 & 11 \\
\hline \multirow{2}{*}{ Buena Vista } & $\mathrm{Pb}-25$ & 289375 & 342550 & 14,70 & 16 \\
\hline & $\mathrm{Pb}-26$ & 289560 & 342460 & 15,00 & 16 \\
\hline Total & 16 & & & 178,4 & 177 \\
\hline
\end{tabular}

Tabla 4. Tipos de muestreo y cantidad total de muestras tomadas.

\begin{tabular}{lcccc}
\hline \multicolumn{1}{c}{ Sector } & Surco & Testigo & Itinerarios & Total \\
\hline Agustina & 12 & 24 & 12 & 48 \\
Yagruma & 15 & 32 & 15 & 62 \\
Prado - Los Hoyos & 14 & 23 & 14 & 51 \\
Mendieta - Banks & 7 & 18 & 7 & 32 \\
Montaña & - & - & - & - \\
San Federico & 21 & 22 & 21 & 64 \\
Buena Vista & 39 & 32 & 39 & 110 \\
& - & - & 62 & 62 \\
Total & $\mathbf{1 0 8}$ & $\mathbf{1 5 1}$ & $\mathbf{1 7 0}$ & $\mathbf{4 2 9}$ \\
\hline
\end{tabular}

La cantidad total de análisis (429) tuvo como objetivo alcanzar la mayor representatividad posible, de modo que se lograra evaluar las potencialidades de los distintos sectores y sin sobrepasar las posibilidades económicas del proyecto inicial. De igual forma obtener una calidad acorde con la etapa de la investigación y los objetivos generales planteados desde el inicio de la misma (Tabla 5). 
Tabla 5. Cantidad de análisis realizados.

\begin{tabular}{cc}
\hline Tipo de análisis & Cantidad \\
\hline Análisis químico para Au por AA & 437 \\
Análisis geoquímico por ICP-MS & 311 \\
\hline
\end{tabular}

\section{Resultados}

Los análisis realizados permitieron revelar contenidos de oro con valores de hasta $2,24 \mathrm{~g} / \mathrm{t}$ en el yacimiento Yagruma; y hasta $2,22 \mathrm{~g} / \mathrm{t}$ en el yacimiento Buena Vista. Los valores de la concentración de plata fueron muy bajos $(<1 \mathrm{~g} / \mathrm{t})$ en todos los sectores estudiados. A continuación, describimos cada uno de los sectores y los resultados obtenidos.

\section{Sector Agustina}

En este sector se tomaron 8 muestras de superficie (Tabla 4) y se realizaron 3 pozos de perforación (Tabla 3, Figura 3). En las muestras de superficie tomadas en las zonas oxidadas se obtuvieron resultados bajos de $\mathrm{Au}$, que varía entre 0,01 y $0,05 \mathrm{~g} / \mathrm{t}$. Se realizaron 3 pozos situados en las partes más elevadas de los cuerpos oxidados, con el objetivo de estudiar la potencia de la zona de oxidación, los mismos tuvieron una profundidad promedio de 9,6 $\mathrm{m}$ dando resultados alentadores desde el comienzo hasta el final con valores entre 0,05 y $0,38 \mathrm{~g} / \mathrm{t}$ (Figura 3 ).

Este último resultado corresponde a una muestra al final del pozo $\mathrm{Pa}-4$ por lo que es de esperar a profundidades mayores, más cerca de los cuerpos minerales, contenidos similares o mayores a estos (Figura 3). La perspectividad del sector en la que se fundamentaría la continuidad de los trabajos se basa en las dimensiones de las zonas de oxidación y en los valores de Au descritos.

Tabla 6. Resultados de los análisis de oro en zonas oxidadas.

\begin{tabular}{cccccccc}
\hline & $\mathbf{A u}$ & $\mathbf{A s}$ & $\mathbf{G a}$ & $\mathbf{H g}$ & $\mathbf{N i}$ & $\mathbf{C o}$ & $\mathbf{F e} \%$ \\
\hline $\mathbf{A u}$ & 1,00 & & & & & & \\
$\mathbf{A s}$ & $\mathbf{0 , 6 4}$ & 1,00 & & & & & \\
$\mathbf{G a}$ & 0,29 & 0,57 & 1,00 & & & & \\
$\mathbf{H g}$ & 0,28 & 0,44 & 0,28 & 1,00 & & & \\
$\mathbf{N i}$ & $-0,37$ & $-0,34$ & $-0,30$ & $-0,08$ & 1,00 & & \\
$\mathbf{C o}$ & $-0,37$ & $-0,31$ & $-0,08$ & $-0,02$ & 0,40 & 1,00 & \\
$\mathbf{F e} \%$ & $\mathbf{0 , 6 9}$ & $\mathbf{0 , 7 1}$ & $\mathbf{0 , 9 2}$ & 0,34 & $-0,50$ & $-0,35$ & 1,00 \\
\hline
\end{tabular}

\section{Sector Yagruma}

En el sector Yagruma se encuentra el prospecto del mismo nombre. En ella se encuentran varios cuerpos minerales de buzamiento abrupto con potencia entre 0,5 y 2,5 m (Kulikov, 1971). El sector cuenta con una amplia zona de oxidación o gossan. En el área de este prospecto se observa una zona de amplio desarrollo de vetas y vetillas de limonita dentro de los límites de la zona de oxidación. Las rocas presentan fuerte hematización. La zona oxidada tiene una extensión de unos $800 \mathrm{~m}$ por el rumbo y unos $200-250 \mathrm{~m}$ por el buzamiento. Es de destacar en este sector que las muestras de superficie dieron contenidos significativos en el orden de 0,04 hasta $1,73 \mathrm{~g} / \mathrm{t}$ de $\mathrm{Au}$, así como valores anómalos de As de hasta $4153 \mathrm{~g} / \mathrm{t}$; se realizaron 3 pozos, 2 de los cuales cortaron la zona oxidada con contenidos muy interesantes de $\mathrm{Au}$, en orden de 0,05 hasta $2,24 \mathrm{~g} / \mathrm{t}$ y As de 20 hasta $1164 \mathrm{~g} / \mathrm{t}$. 


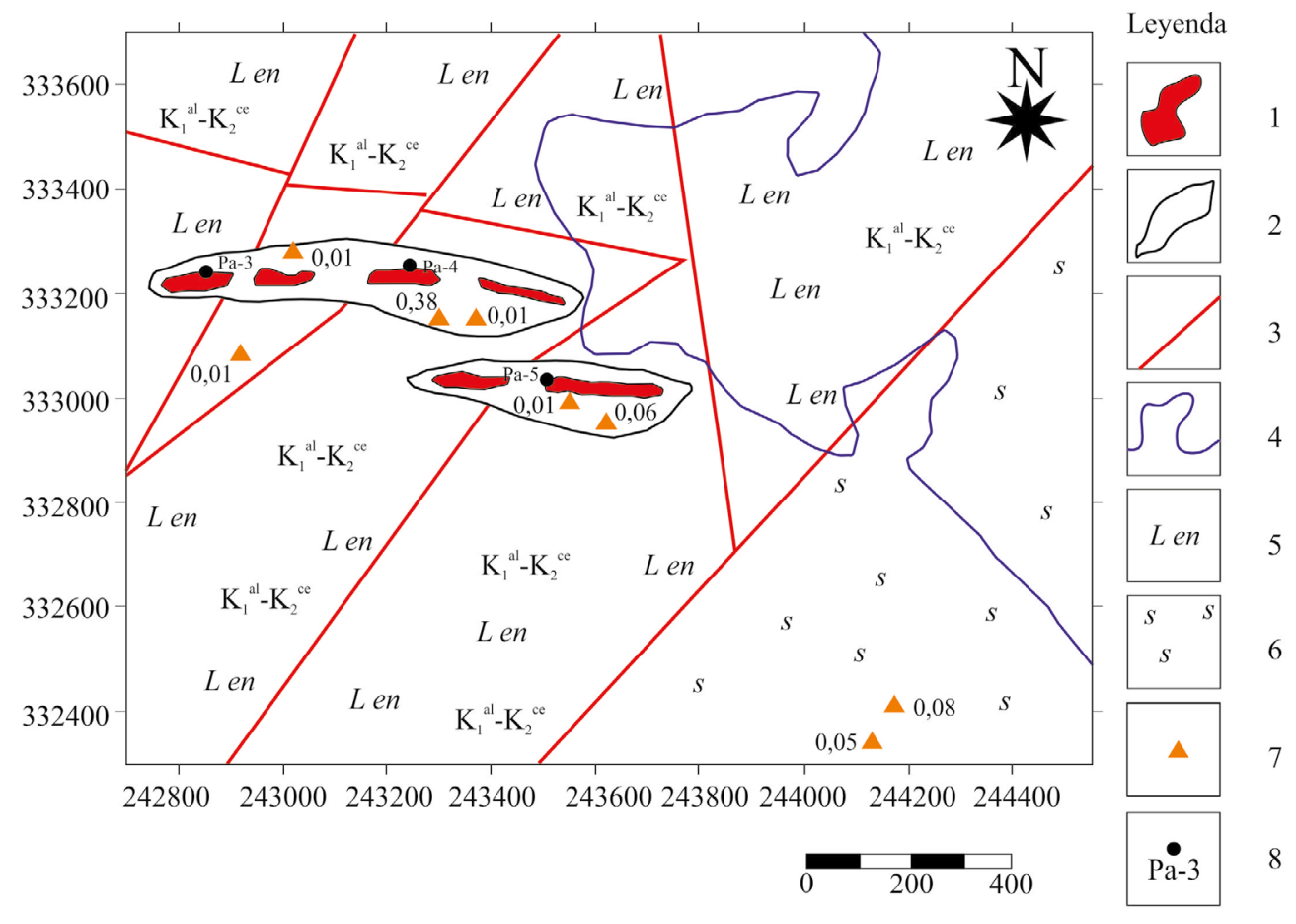

Figura 3. Mapa geológico y de datos reales del sector Agustina. Escala gráfica en m. 1. Cuerpos minerales. 2. Cuerpos de diabasa. 3. Fallas. 4. Río Tortuga. 5. Formación Encrucijada. 6. Serpentinitas del Complejo Ofiolítico. 7. Punto de muestreo de oro g/t. 8. Pozo de perforación.

El otro pozo realizado se ubicó fuera de la zona de 4), y el mismo cortó mineralización de Au en el orden oxidación, a unos $280 \mathrm{~m}$ del pozo Py-9 al SE (Figura de 0,05-0,07 g/t hasta una profundidad de 6,30 m.
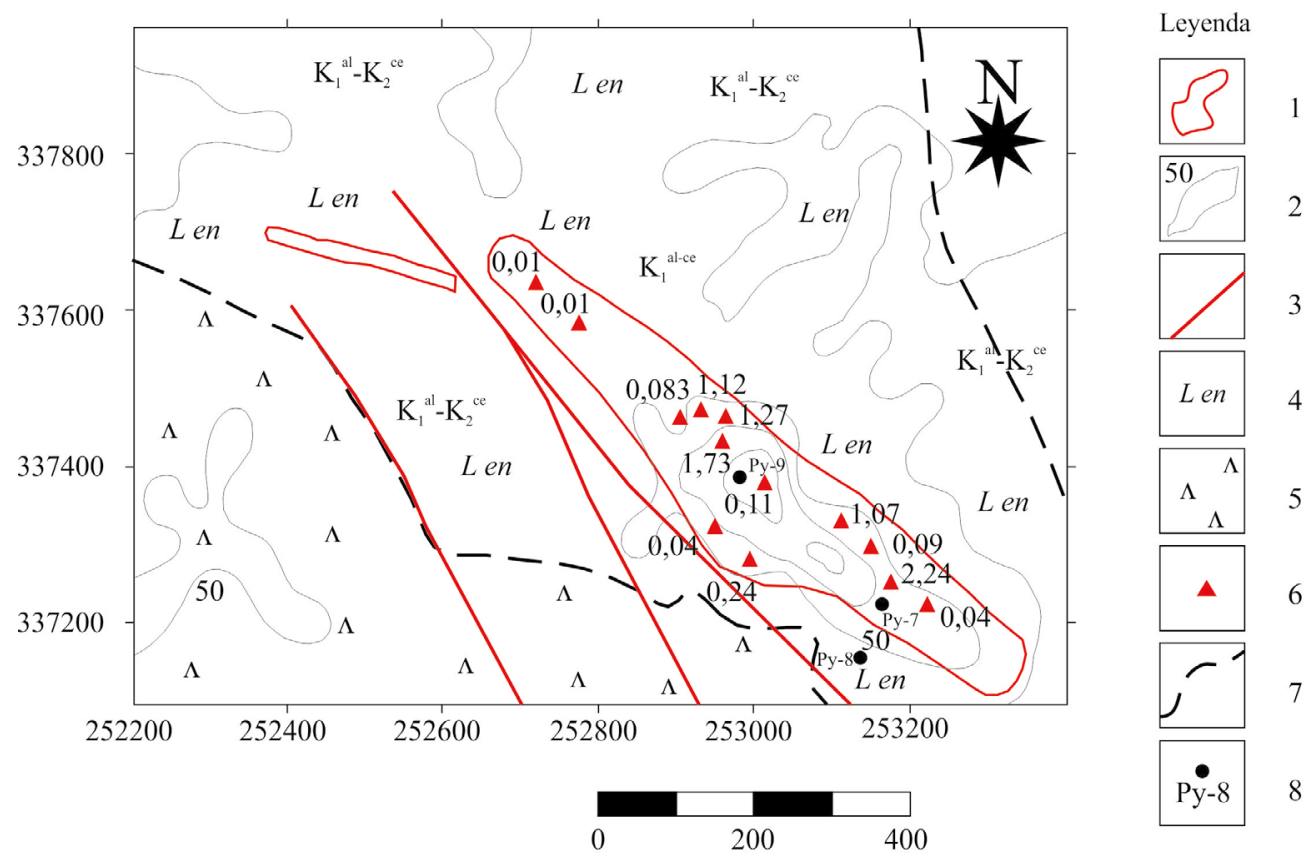

Figura 4. Mapa geológico y de datos reales del sector Yagruma. Escala gráfica en m. 1. Zona de oxidación. 2. Curvas de nivel. 3. Fallas. 4. Formación Encrucijada. 5. Gabros - diabasa del Complejo Ofiolítico. 6. Toma de muestra g/t. 7. Contacto litológico. 8. Pozo de perforación. 
Se puede ver que existe una ligera correlación entre los valores de $\mathrm{Au}-\mathrm{As}\left(\mathrm{r}^{2}=0,64\right), \mathrm{Au}-\mathrm{Fe}\left(\mathrm{r}^{2}=0,69\right)$ y a su vez hay una correlación fuerte entre Fe y As $\left(\mathrm{r}^{2}=0,71\right)$ y entre el Ga y Fe $\left(r^{2}=0,92\right)$, que evidencia una posible asociación entre estos elementos, y relaciones débiles entre $\mathrm{Au}$ y Ni - Co (Tabla 6).

\section{Sector Prado - Los Hoyos}

En esta área se muestrearon todas las zonas de oxidación, arrojando resultados interesantes en superficie, en el orden de 0,03-0,43 g/t de Au (Figura 5). Se realizaron dos pozos, que no aportaron resultados significativos, pero permitieron comprobar el acuñamiento de la zona de oxidación hacia el noroeste.

En el caso del pozo Pp-12, las muestras dieron contenidos de $0,03-0,07 \mathrm{~g} / \mathrm{t}$ de $\mathrm{Au}$, lo que puede indicar que los mayores contenidos estén ubicados en las partes más oxidadas de menor elevación.

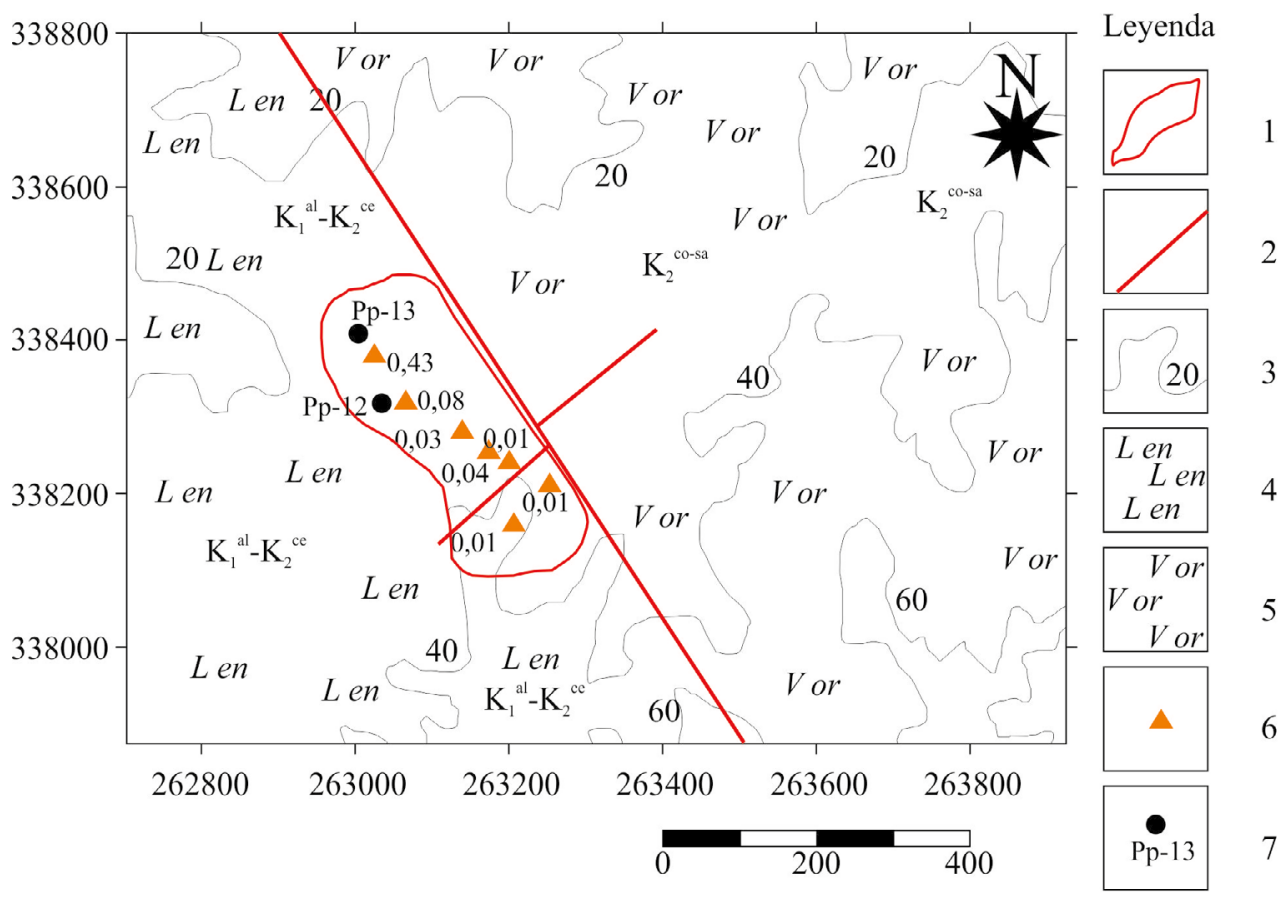

Figura 5. Mapa geológico y de datos reales del sector Prado - Los Hoyos. Escala gráfica en m. 1. Zona de oxidación. 2. Fallas. 3. Curvas de nivel. 4. Formación Encrucijada. 5. Formación Orozco. 6. Muestreo de oro g/t. 7. Pozo de perforación.

\section{Sector Mendieta - Banks}

La zona de oxidación se caracteriza por la presencia de vetillas de cuarzo. En este sector se tomaron 7 muestras de superficie y se realizaron 2 pozos de perforación. En las muestras de superficie tomadas en las zonas oxidadas, localizadas en basaltos oxidados, costras limonítico - hematíticas, se obtuvieron resultados en el orden de 0,03 hasta $0,46 \mathrm{~g} / \mathrm{t}$ de Au.
Los 2 pozos realizados con una profundidad promedio de $8,9 \mathrm{~m}$ aportaron resultados interesantes en los primeros metros con contenidos de hasta $1,43 \mathrm{~g} / \mathrm{t}$ de $\mathrm{Au}$, aunque después en profundidad los contenidos disminuyen en el orden de 0,03-0,06 g/t (Figura 6). 


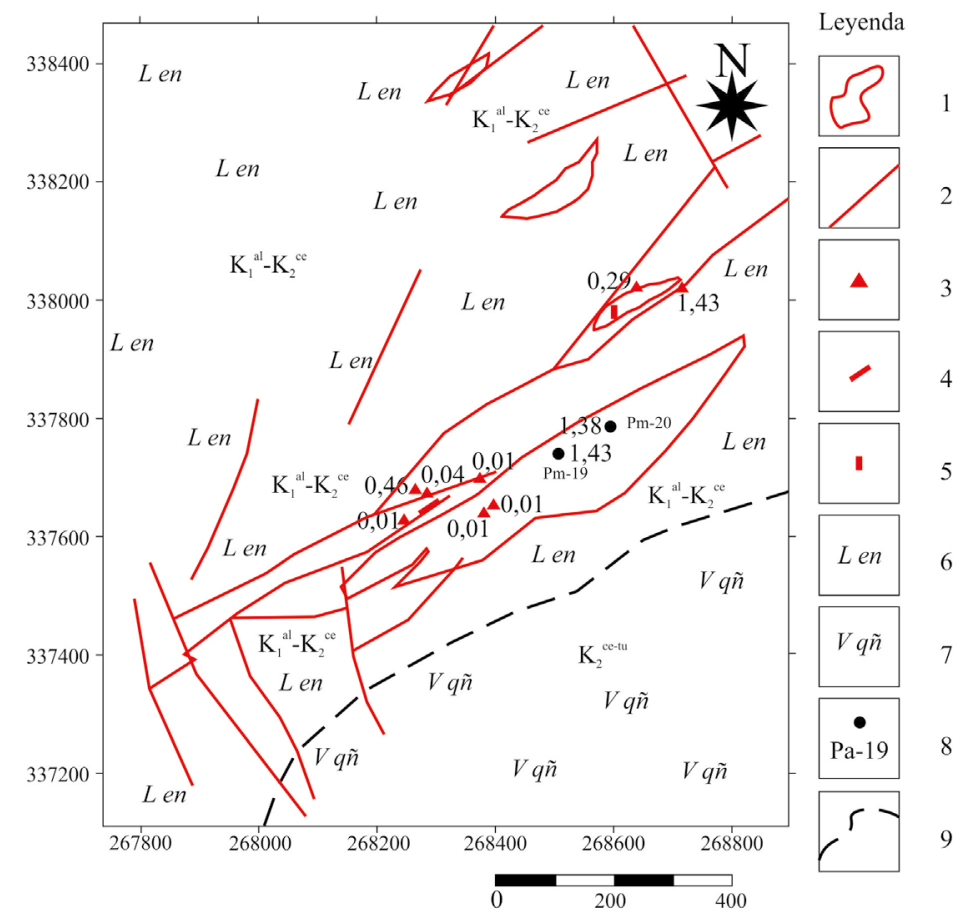

Figura 6. Mapa geológico y de datos reales del sector Mendieta - Bank. Escala gráfica en m. 1. Zona de oxidación. 2. Fallas. 3. Muestra de afloramiento g/t. 4. Socavón. 5. Pozo de minas. 6. Formación Encrucijada. 7. Formación Quiñones. 8. Pozos de perforación. 9. Contacto litológico.

\section{Sector Montaña}

En este prospecto se tomaron un total de 13 muestras de afloramientos y se realizaron 2 pozos de perforación (Figura 7). Las muestras tomadas en superficie dieron valores entre $0,03-0,62 \mathrm{~g} / \mathrm{t}$ de $\mathrm{Au}$.

En profundidad en el pozo Po-22, situado cerca de una antigua trinchera de prospección y hasta los 11,70 m se obtuvieron resultados del orden de $0,03-0,35$ $\mathrm{g} / \mathrm{t}$ de $\mathrm{Au}$, este último contenido ubicado entre 4,30 $4,80 \mathrm{~m}$. En el pozo Po-27, ubicado a unos $200 \mathrm{~m}$ del Po-22. En la misma zona de alteración hidrotermal, hasta una profundidad de $12,0 \mathrm{~m}$ se registran valores muy estables, entre $0,03-0,08 \mathrm{~g} / \mathrm{t}$ de Au que, aunque bajos no dejan de ser interesantes como guía para la prospección.

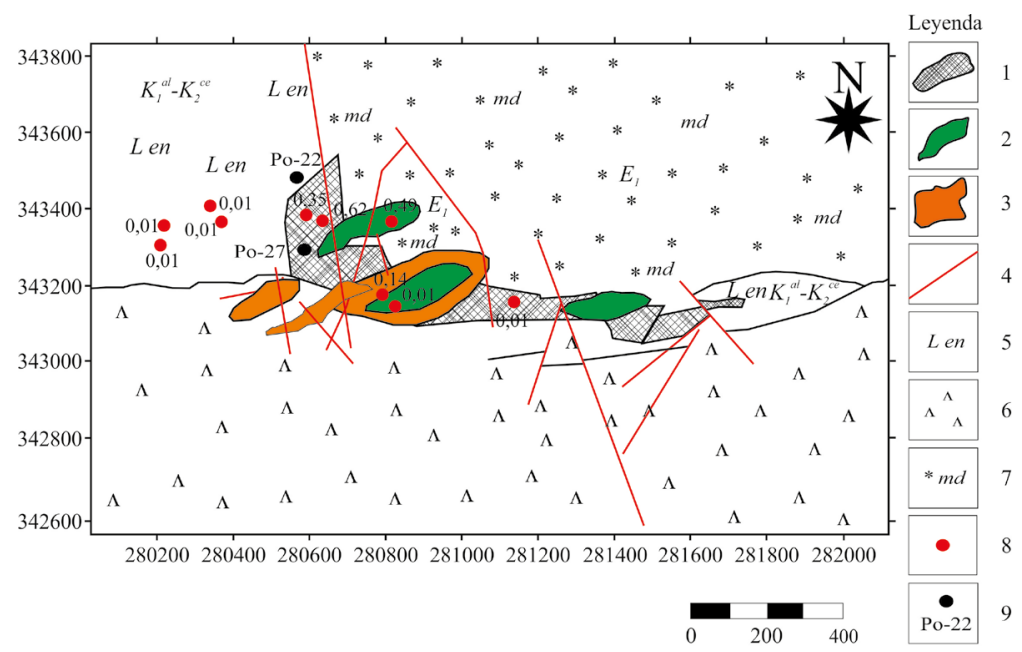

Figura 7. Mapa geológico y de datos reales sector Montaña. Escala gráfica en m. 1. Alteración hidrotermal. 2. Anomalía geoquímica de $\mathrm{Zn}$. 3. Anomalía geoquímica de $\mathrm{Cu}$. 4. Fallas. 5. Formación Encrucijada. 6. Gabro - diabasa del Complejo Ofiolítico. 7. Formación Madruga. 8. Muestreo de oro g/t. 9. Pozo de perforación. 


\section{Sector San Federico}

La existencia de minas antiguas, zonas de alteración hidrotermal, mineralización sulfurosa y las anomalías geofísicas - geoquímicas presentes en esta área eleva la potencialidad de este sector. Como se puede observar
(Tabla 7) existe una correlación buena entre el $\mathrm{Au}-\mathrm{Hg}$ $\left(\mathrm{r}^{2}=0,83\right)$ y As y $\mathrm{Hg}\left(\mathrm{r}^{2}=0,79\right)$ y una ligera correlación entre el $\mathrm{Au}-\mathrm{As}\left(\mathrm{r}^{2}=0,62\right)$. Es bueno destacar que en este sector fue donde los valores de Hg fueron los más elevados, en el orden de $0,25-9,2 \mathrm{~g} / \mathrm{t}$.

Tabla 7. Matriz de correlación ( $\mathrm{n}=43$ ).

\begin{tabular}{llllllll}
\hline & $\mathbf{A u}$ & $\mathbf{A s}$ & $\mathbf{C u}$ & $\mathbf{S b}$ & $\mathbf{Z n}$ & $\mathbf{P b}$ & $\mathbf{H g}$ \\
\hline $\mathbf{A u}$ & 1,00 & & & & & & \\
As & $\mathbf{0 , 6 2}$ & 1,00 & & & & & \\
$\mathbf{C u}$ & $-0,11$ & 0,03 & 1,00 & & & & \\
Sb & $-0,18$ & 0,07 & 0,66 & 1,00 & & & \\
$\mathbf{Z n}$ & $-0,04$ & 0,09 & 0,32 & 0,29 & 1,00 & & \\
$\mathbf{P b}$ & 0,07 & 0,39 & 0,66 & 0,76 & 0,26 & 1,00 & \\
$\mathbf{H g}$ & $\mathbf{0 , 8 3}$ & $\mathbf{0 , 7 9}$ & $-0,09$ & $-0,18$ & $-0,20$ & 0,21 & 1,00 \\
\hline
\end{tabular}

En este prospecto se tomaron un total de 21 muestras de afloramientos y se realizaron 2 pozos de perforación (Figura 8). Las muestras tomadas en superficie dieron valores entre $0,34-1,89 \mathrm{~g} / \mathrm{t}$ de $\mathrm{Au}$, ubicados en las zonas de basaltos con alteración hidrotermal y en afloramientos de bloques limonítico - hematíticos.

En el pozo Ps-23, ubicado a un costado de la antigua torre de caldera, usada en la explotación del yacimiento, hasta una profundidad de $10,00 \mathrm{~m}$ se registran resultados entre $0,03-0,82 \mathrm{~g} / \mathrm{t}$ de $\mathrm{Au}$, este último valor en el primer metro de sondeo. El otro pozo Ps-24 a aproximadamente $100 \mathrm{~m}$ del anterior, revela también en los primeros metros valores significativos de $0,14 \mathrm{~g} / \mathrm{t}$ de $\mathrm{Au}$. Es de destacar que al no poderse instalar la perforadora en las partes más elevadas del relieve (yacimiento), no se pudo precisar la potencia de la zona de oxidación y su variabilidad.

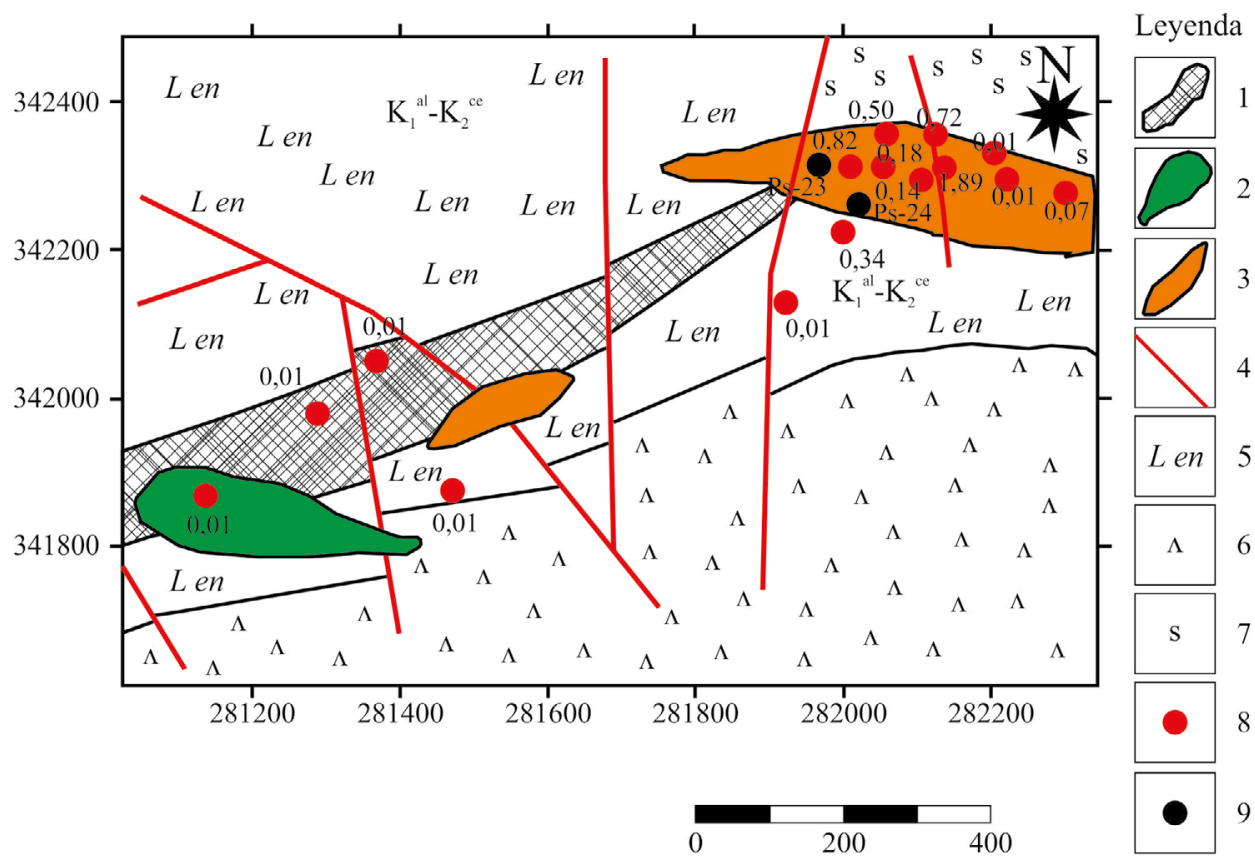

Figura 8. Mapa geológico y de datos reales sector San Federico. Escala gráfica en m. 1. Alteración hidrotermal. 2. Anomalía geofísica de PI 30\%. 3. Anomalía geoquímica de cobre. 4. Fallas. 5. Formación Encrucijada. 6. Gabro - diabasa del Complejo Ofiolítico. 7. Serpentinitas del Complejo Ofiolítico. 8. Muestreo de oro g/t. 9. Pozo de perforación. 


\section{Sector Buena Vista}

Teniendo en cuenta el muestreo de superficie, podemos señalar que el área en sentido general es muy interesante para la localización de posible mineralización aurífera, con valores que oscilan entre 0,11 y $2,28 \mathrm{~g} / \mathrm{t}$.

Los análisis de ICP realizados revelaron altos contenidos de los elementos químicos de $\mathrm{Ga}, \mathrm{As}, \mathrm{Sb}$,
Bi y $\mathrm{Hg}$ que coinciden espacialmente con la presencia de contenidos significativos de Au. Podemos observar como existe una ligera correlación entre los valores $\mathrm{Au}-\mathrm{Fe}\left(\mathrm{r}^{2}=0,66\right)$ evidenciando una posible asociación entre estos elementos. También es significativa la correlación existente entre $\mathrm{Mo}-\mathrm{As}, \mathrm{Pb}-\mathrm{Fe}$ y $\mathrm{Cu}-\mathrm{Zn}$ (Tabla 8).

Tabla 8. Matriz de correlación, Sector Buena Vista ( $\mathrm{n}=71)$.

\begin{tabular}{|c|c|c|c|c|c|c|c|c|c|c|c|c|c|}
\hline & $\mathbf{A u}$ & $\mathbf{A g}$ & As & $\mathbf{C u}$ & Sb & $\mathbf{Z n}$ & $\mathbf{P b}$ & $\mathrm{Fe} \%$ & Ba & Co & $\mathrm{Cr}$ & Mo & $\mathbf{T i}$ \\
\hline Au & 1,00 & & & & & & & & & & & & \\
\hline $\mathbf{A g}$ & $-0,10$ & 1,00 & & & & & & & & & & & \\
\hline As & 0,49 & 0,38 & 1,00 & & & & & & & & & & \\
\hline $\mathrm{Cu}$ & $-0,19$ & $-0,34$ & 0,04 & 1,00 & & & & & & & & & \\
\hline Sb & 0,02 & $-0,04$ & $-0,26$ & $-0,20$ & 1,00 & & & & & & & & \\
\hline $\mathbf{Z n}$ & $-0,07$ & $-0,26$ & $-0,11$ & $\mathbf{0 , 8 0}$ & $-0,02$ & 1,00 & & & & & & & \\
\hline $\mathbf{P b}$ & $\mathbf{0 , 5 5}$ & 0,52 & 0,63 & $-0,10$ & 0,09 & $-0,19$ & 1,00 & & & & & & \\
\hline $\mathrm{Fe} \%$ & 0,66 & 0,14 & 0,43 & 0,07 & $-0,04$ & $-0,14$ & $\mathbf{0 , 8 5}$ & 1,00 & & & & & \\
\hline Ba & 0,41 & 0,03 & $-0,06$ & $-0,21$ & 0,35 & $-0,12$ & 0,27 & 0,22 & 1,00 & & & & \\
\hline Co & $-0,06$ & $-0,41$ & $-0,04$ & 0,29 & $-0,15$ & 0,07 & $-0,20$ & $-0,11$ & $-0,29$ & 1,00 & & & \\
\hline $\mathrm{Cr}$ & $-0,03$ & $-0,03$ & $-0,16$ & $-0,12$ & $-0,19$ & $-0,09$ & $-0,31$ & $-0,25$ & $-0,20$ & 0,50 & 1,00 & & \\
\hline Mo & $-0,25$ & 0,53 & $\mathbf{0 , 8 7}$ & 0,40 & $-0,15$ & 0,12 & 0,41 & 0,26 & $-0,32$ & 0,11 & $-0,18$ & 1,00 & \\
\hline $\mathbf{T i}$ & 0,05 & $-0,02$ & $-0,17$ & $-0,18$ & $-0,12$ & $-0,07$ & $-0,25$ & $-0,20$ & 0,17 & $-0,22$ & $-0,16$ & $-0,28$ & 1,00 \\
\hline
\end{tabular}

A continuación, se exponen los resultados más significativos encontrados en las 2 elevaciones que componen el antiguo depósito Buena Vista y en las lomas 3 y 4 . Todos los valores están expresados en $\mathrm{g} / \mathrm{t}$.

- Loma 1 (escombrera) muestra B - $017 \mathrm{Au}-1,14$

- Loma 2 (escombrera) muestra B - $0173 \mathrm{Au}-0,51$, As $-258, \mathrm{Sb}-<2, \mathrm{Ga}-46$

- Loma 3 muestra B - $0164 \mathrm{Au}-1,94$, As - 1180, $\mathrm{Sb}-19, \mathrm{Ga}-66$

- Loma 4 muestra B - $0146 \mathrm{Au}-1,14$, As - 465, $\mathrm{Sb}-<2, \mathrm{Bi}-1462, \mathrm{Ga}-25$ muestra B -0150 $\mathrm{Au}-2,22, \mathrm{As}-1726, \mathrm{Sb}-94, \mathrm{Bi}-1062, \mathrm{Ga}-63$, $\mathrm{Hg}-2,4$

Mediante los sondeos se reveló que el pozo $\mathrm{Pb}-25$ (14,70 $\mathrm{m}$ de prof.) cortó una zona de intensa alteración hidrotermal, con mineralización semi - masiva de sulfuros de 9,90 - 11,90 m, registrándose los valores más elevados de Au, que van desde 0,38 hasta 0,47 $\mathrm{g} / \mathrm{t}$. El pozo $\mathrm{Pb}-26$ perforado hasta $15,0 \mathrm{~m}$ cortó una zona de basaltos oxidados, obteniéndose los valores más significativos entre 0,10 y $3,40 \mathrm{~m}$ con contenidos de 0,04 a $0,31 \mathrm{~g} / \mathrm{t}$. También es destacable que en los trabajos de exploración realizados durante 1987 en Buena Vista se conoce que, a una profundidad de 60,0 $\mathrm{m}$, en la intersección del cuerpo mineral, una muestra registró un contenido de 2,35 g/t Au. Los resultados anteriormente expuestos permiten corroborar la alta perspectividad de todo este sector, no solo por los contenidos significativos de $\mathrm{Au}$ (de hasta $2,22 \mathrm{~g} / \mathrm{t}$ ), sino también por los valores anómalos de los elementos indicadores de Au (Figura 9). 


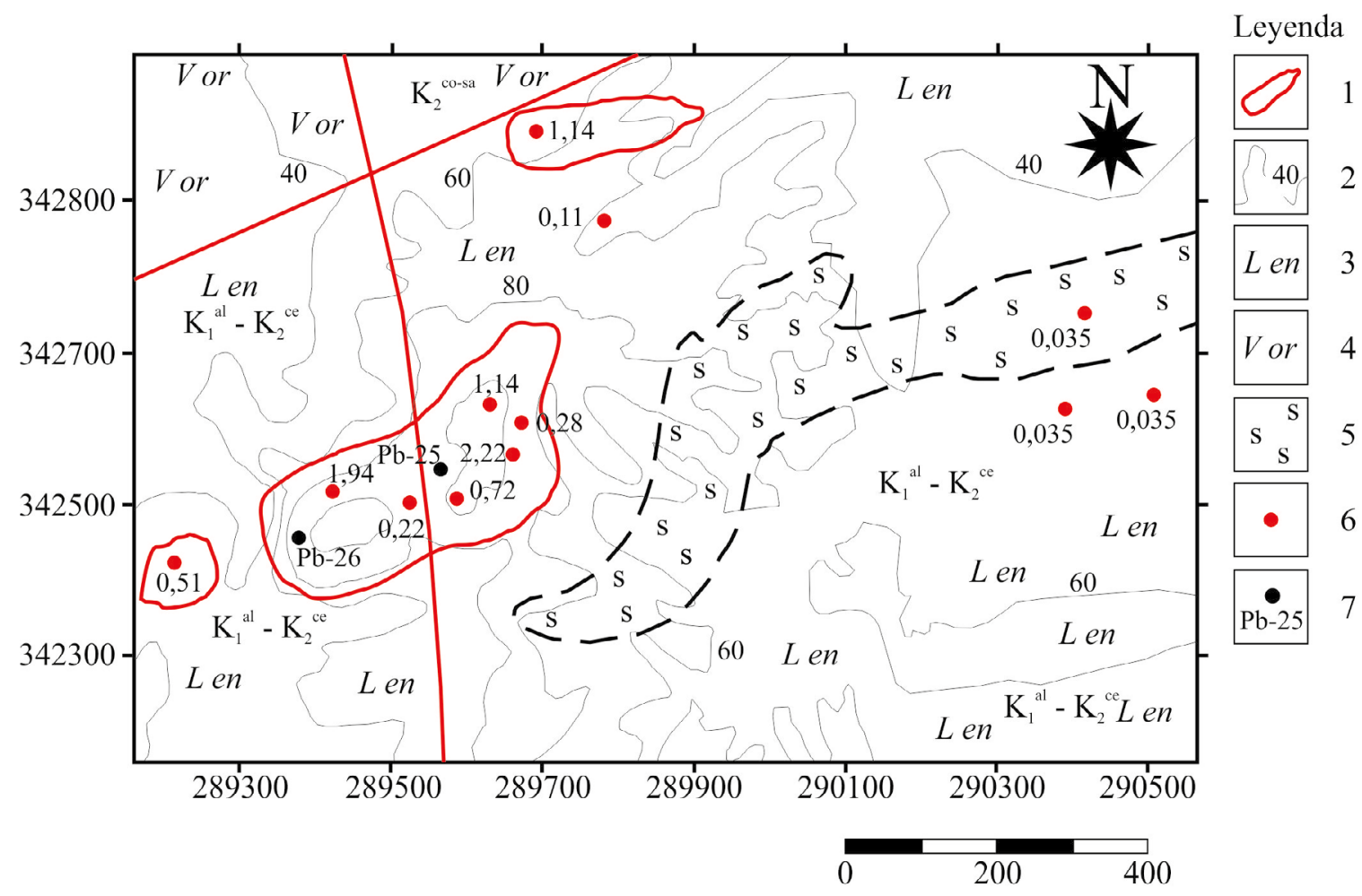

Figura 9. Mapa geológico y de datos reales sector Buena Vista. Escala gráfica en m. 1. Zona de oxidación. 2. Curvas de nivel. 3. Formación Encrucijada. 4. Formación Orozco. 5. Serpentinitas del Complejo Ofiolítico. 6. Muestreo de oro g/t. 7. Pozo de perforación.

\section{Análisis de los resultados}

En la región se encontraron áreas con concentraciones elevadas de $\mathrm{Sb}, \mathrm{As}, \mathrm{Ga}, \mathrm{Hg}, \mathrm{Bi}, \mathrm{Cu}, \mathrm{Zn}, \mathrm{Pb}, \mathrm{Ba}, \mathrm{Fe}$ y Be. La presencia de estos elementos está vinculada con la composición químico - mineralógica de la mineralización primaria en los diferentes yacimientos y prospectos evaluados. Así, por ejemplo, se aprecian los mayores contenidos de estos elementos en los siguientes sectores:

As - (Agustina, Yagruma, Montaña, San Federico y Buena Vista)

Bi - (Yagruma, Prado - Los Hoyos y Buena Vista)

$\mathrm{Sb}$ - (Agustina, Yagruma, Prado - Los Hoyos y Buena Vista)

Ga - (Agustina, Yagruma, Prado - Los Hoyos y Buena Vista)

$\mathrm{Hg}$ - (Yagruma, Prado - Los Hoyos, San Federico)

Los contenidos de oro obtenidos de las muestras, tanto de afloramiento como de perforación, oscilan entre 0,01 y 2,24 g/t. En todas las zonas evaluadas se determinó la presencia de este metal. Los mayores contenidos se obtuvieron en los sectores Yagruma, San Federico y Buena Vista. Las asociaciones geoquímicas naturales reveladas en el área están vinculadas con estos sectores de mayor interés, así tenemos:

- $\mathrm{As}-\mathrm{Bi}-\mathrm{Ga}-\mathrm{Hg}$. Sector Yagruma y Buena Vista.

- $\mathrm{As}-\mathrm{Cu}-\mathrm{Ti}-\mathrm{Hg}$. Sector San Federico.

En las zonas de oxidación de todos los sectores se comprobó la presencia de contenidos interesantes de oro que oscilan entre 0,05 y $2,24 \mathrm{~g} / \mathrm{t}$. Atendiendo a los estimados de tonelaje y contenido medio de oro, es posible identificar algunos sectores más interesantes que otros, aunque como ya señalamos el grado de certidumbre no es alto al ser recursos hipotéticos, como son: Yagruma, donde se concentra el 30,11\% de los recursos de oro, San Federico con un 20,59\% de los recursos y Buena Vista con el 26,61\%. Estos tres sectores representan el $77,31 \%$ del total de los recursos de la región.

El volumen más grande corresponde (Tabla 10) con el sector San Federico con $5400000 \mathrm{~m}^{3}$, a continuación, se encuentra Montaña con $5400000 \mathrm{~m}^{3}$, Buena Vista 
con un total de $4200000 \mathrm{~m}^{3}$ y finalmente Yagruma con $3458250 \mathrm{~m}^{3}$. Es precisamente en estos sectores donde los contenidos medios de Au son más interesantes.

El coeficiente $r^{2}$ puede variar de -1 a 1 , donde el signo indica la dirección de la correlación y el valor numérico, la magnitud de la correlación. En este contexto en los sectores Yagruma, Montaña y San Federico en todos los casos la correlación es positiva, lo que indica que cuando un valor aumenta, el otro también y viceversa. Existe correlación positiva considerable entre el oro y el arsénico en los sectores Yagruma y Montaña, debido a la afinidad geoquímica (Vologdin y Stepanov, 1967; Kulikov, 1971; Cazañas-Díaz et al., 2017) que tienen estos elementos. De igual forma sucede entre el oro y el hierro y el arsénico y el hierro en Yagruma. En el sector Montaña también existe una correlación positiva considerable entre el oro y el mercurio y el arsénico y mercurio. Mientras que en San Federico la correlación entre el oro y el arsénico es positiva media, al igual que con el $\mathrm{Pb}$ y Fe. Por su parte el cobre presenta una correlación positiva considerable con el $\mathrm{Zn}$, al igual que el $\mathrm{Pb}$ y el Fe.

\section{Cálculo de recursos}

La clasificación de los recursos en el trabajo se ha realizado acorde con las clasificaciones que actualmente publica el Servicio Geológico de los Estados Unidos en su resumen estadístico del Mineral Commodity Summaries (U.S. Geological Survey, 2020) y la misma parte de modelos genéticos y de la clasificación de los recursos y reservas de minerales útiles sólidos aprobado por el Ministerio de la Industria Básica (actualmente Ministerio de Energía y Minas) y puesto en vigor en la Oficina Nacional de Recursos Minerales (ONRM) en 1999. Teniendo en cuenta las características geológicas de la región, el grado de conocimiento que de ella se tiene, la historia metalogénica de la misma, es que se calculó, para todos los sectores, recursos hipotéticos, esto es, los recursos sin descubrir que son parecidos a cuerpos minerales conocidos y que pueden estar razonablemente supuestos de existir en igual distrito o región bajo condiciones geológicas análogas. Si la exploración confirma su existencia y denuncia bastante información acerca de su calidad, grado, y cantidad, pudieran ser reclasificadas como recursos identificados.

Tabla 9. Condiciones industriales empleadas para el cálculo de los recursos.

\begin{tabular}{clc}
\hline No. & \multicolumn{1}{c}{ Parámetro para el cálculo } & Condición mínima \\
\hline 1 & Contenido mínimo industrial & $1,0 \mathrm{~g} / \mathrm{t}$ \\
2 & Cut off grade & $0,3 \mathrm{~g} / \mathrm{t}$ \\
3 & Metro - gramo & 0,3 \\
4 & Potencia máxima de intercalación de estériles & $5 \mathrm{~m}$ \\
5 & Potencia mínima industrial & $1 \mathrm{~m}$ \\
\hline
\end{tabular}

En relación con el metro gramo $(\mathrm{mg})$ también utilizado el metro \% $(\mathrm{m} \%)$, en función de si la ley se mide en $\%$ o en gramos por toneladas, el mismo se utiliza para delimitar los sectores poco potentes, pero ricos en el componente útil y no es otra cosa que el cálculo de la potencia mínima industrial por el contenido mínimo industrial o de los bordes. Cuando los sondeos que cortan la mineralización, no cumplen con la potencia mínima industrial, pero tienen una alta ley, entonces se calcula un metro por ciento o metro gramo promedio a partir del cual se van a comparar los sondeos o parte de los mismo que cumplan ese requisito (Lepin y Ariosa, 1986).

Los parámetros empleados (Tabla 9) para el cálculo de los recursos fueron seleccionados por analogía con el yacimiento tipo sedex Oro Castellanos, ubicado en el campo mineral Santa Lucía - Castellanos, al NO de Pinar del Río, mientras que el contenido medio se tomó, teniendo en cuenta que es equivalente al cut off grade utilizado en el contorneo de la reserva de oro del yacimiento Castellanos y Cobre Mantua en Pinar del Río y Golden Hill en Camagüey. El peso volumétrico se tomó por analogía basándonos en los diferentes trabajos realizados con anterioridad en la región como los cálculos de reservas de cobre en los yacimientos Cacarajícara, Yagruma y Buena Vista. En el caso del coeficiente de confiabilidad estos valores se aproximan al rango de valores de coeficientes propuestos internacionalmente (Escobar, 1973; Estévez-Cruz, 2000; Pérez-Vázquez, 2000; Cazañas-Díaz et al., 2017).

Para el cálculo de los recursos los parámetros utilizados se obtuvieron, para cada uno de los sectores, de la siguiente forma: 
Área (S): se calculó mediante el método de la red milimétrica dentro de los límites supuestos de la zona de oxidación, teniendo en cuenta la escala del mapa geológico y se contorneó las zonas de oxidación.

Potencia media (H): para la determinación de la potencia media se tuvo en cuenta la información de los trabajos anteriores en los diferentes sectores. Estos sondeos, aunque no fueron realizados con el objetivo de estudiar la zona de oxidación, pero si brindan la potencia de la misma.

Contenido medio (C): se tomaron los contenidos de oro en sondeos de los trabajos de reconocimiento realizados recientemente. Se promediaron los valores de Au mayores o igual que 0,3 g/t. Se consideró el valor mínimo 0,3 g/t Au.

Peso volumétrico (D): partiendo de que se estaba en fase de trabajos de reconocimiento geológico, no ha sido posible determinar la masa volumétrica, por lo cual la misma se tomó por analogía como $2,6 \mathrm{t} / \mathrm{m}^{3}$.

Coeficiente de confiabilidad (K): se determina teniendo en cuenta la relación entre el área mineralizada y el área del sector de trabajo que, para nuestro caso, coincide con los límites de la zona de oxidación. Sin embargo, como no es posible con la información existente definir la zona mineralizada, se consideró que el coeficiente de confiabilidad es de 0,2 , es decir, que el
$20 \%$ del área de la zona de oxidación está mineralizada, este coeficiente de 0,2 se utilizó para Yagruma y Buena Vista ya que las muestras tomadas tienen una distribución más regular, los restante sectores se tomó 0,1 y 0,15 . Estos valores se aproximan al rango de valores de coeficientes propuestos internacionalmente $(10-20 \%)$ para esta categoría de recursos con una óptica más conservadora, para no sobreestimar los resultados, ya que el comportamiento del oro está muy influenciado por diferentes factores geológicos, físico - químicos y climáticos que pueden alterar los resultados.

Finalmente, para el cálculo se utilizó la fórmula siguiente:

Qc $=$ K x S x H x Cx D donde:

Qc - Recursos

$\mathrm{K}$ - Coeficiente de confiabilidad del pronóstico

$\mathrm{S}$ - Área de distribución de las zonas de oxidación

$\mathrm{H}$ - Potencia media del pronóstico

$\mathrm{C}$ - Contenido medio del componente útil

$\mathrm{D}$ - Peso volumétrico promedio

Como se puede apreciar en la Tabla 10 al concluir los cálculos, los recursos hipotéticos totales de los 7 sectores, representan un total de 7,14 t de $\mathrm{Au}$ (229556,355 oz/troy).

Tabla 10. Cálculo de los recursos.

\begin{tabular}{ccccccccc}
\hline Sector & $\begin{array}{c}\text { Área } \\
\left(\mathbf{m}^{2}\right)\end{array}$ & $\begin{array}{c}\text { Poten. } \\
(\mathbf{m})\end{array}$ & $\begin{array}{c}\text { Volumen } \\
\left(\mathbf{m}^{\mathbf{3}}\right)\end{array}$ & $\begin{array}{c}\text { Peso } \\
\mathbf{v o l u m} \\
\left(\mathbf{t} / \mathbf{m}^{\mathbf{3}}\right)\end{array}$ & $\begin{array}{c}\text { Tonelaje } \\
\mathbf{d e} \\
\mathbf{m e n a}(\mathbf{t})\end{array}$ & $\begin{array}{c}\text { Coefic. de } \\
\mathbf{c o n f i a b}\end{array}$ & $\begin{array}{c}\text { Cont. } \\
\text { medio } \\
(\mathbf{g} / \mathbf{t})\end{array}$ & $\begin{array}{c}\text { Cantidad } \\
\text { de metal } \\
\mathbf{A u}(\mathbf{t})\end{array}$ \\
\hline Agustina & 147500 & 15 & 2212500 & 2,60 & 5752500 & 0,1 & 0,4 & 0,23 \\
Yagruma & 230550 & 15 & 3458250 & 2,60 & 8991450 & 0,2 & 1,2 & $\mathbf{2 , 1 5}$ \\
Prado & 80000 & 15 & 1200000 & 2,60 & 3120000 & 0,1 & 0,4 & 0,12 \\
Mendieta-Bank & 100000 & 15 & 1500000 & 2,60 & 3900000 & 0,15 & 0,8 & 0,47 \\
Montaña & 280000 & 15 & 4200000 & 2,60 & 10920000 & 0,15 & 0,5 & 0,8 \\
San Federico & 360000 & 15 & 5400000 & 2,60 & 14040000 & 0,15 & 0,7 & $\mathbf{1 , 4 7}$ \\
Buena Vista & 242170 & 15 & 3632550 & 2,60 & 9444630 & 0,2 & 1,0 & $\mathbf{1 , 9}$ \\
\hline & & \multicolumn{7}{c}{ Total } \\
\hline
\end{tabular}

De acuerdo con los resultados obtenidos de las distintas zonas estudiadas y atendiendo a los estimados de tonelaje y contenido medio de $\mathrm{Au}$, resultaron ser los más interesantes, los sectores San Federico, Montaña, Buena Vista y finalmente Yagruma. Estos sectores acumulan el 88,52\% de los recursos de Au de la región. Así tenemos que Yagruma concentra el 30\% de los recursos, Buena Vista el 27\%, San Federico el 21\% y por último Montaña con un $11 \%$. 


\section{Conclusiones}

La existencia de metales preciosos en la región Bahía Honda quedó demostrada sobre la base del muestreo geoquímico realizado en las zonas de oxidación o gossan, donde se pudo determinar la presencia de valores significativos del contenido de $\mathrm{Au}$ en todos los prospectos evaluados, oscilando entre 0,05 y 2,24 $\mathrm{g} / \mathrm{t}$, lo cual permitió realizar el cálculo de los recursos hipotéticos.

De los 7 sectores estudiados, San Federico, Montaña, Buena Vista y Yagruma, resultaron ser los más interesantes atendiendo a los estimados de tonelaje y contenido medio de Au.

El estudio demostró que las áreas más interesantes para la localización de acumulaciones de oro coinciden con las zonas de oxidación de los yacimientos y prospectos minerales ya conocidos.

Los basaltos del complejo ofiolítico y rocas asociadas del arco volcánico Cretácico son las principales litologías contenedoras de las acumulaciones de oro, sobre todo las que se encuentran en la parte superior de la mineralización sulfurosa.

Los recursos hipotéticos de la región Bahía Honda ascienden a 7,14 t de $\mathrm{Au}$, de las cuales el $88,52 \%$ se concentran en los sectores Yagruma (30\%), Buena Vista (27\%), San Federico (21\%) y Montaña (11\%).

Finalmente se arribó a la conclusión que sería prudente pasar a la prospección, en el orden que se especifica, los siguientes sectores: Yagruma, Buena Vista, San Federico y Montaña, con el objetivo de disminuir el grado de incertidumbre acerca de la evaluación de recursos de $\mathrm{Au}$ en la zona estudiada, incrementar los trabajos de exploración y muestreo sistemático de tal manera que se eleve el grado de confianza en los datos obtenidos.

\section{Agradecimientos}

Los autores desean agradecer especialmente a la Empresa Geominera de Pinar del Río, por su ayuda incondicional en la revisión de los materiales de archivos, sin los cuales hubiera sido imposible este trabajo. Al Departamento de Geociencias de la Universidad Tecnológica de La Habana "José Antonio Echeverría" que autorizó realizar los trabajos de campo.

\section{Referencias}

Álvarez, P.; Díaz, G.C.; Barbón, R. (1991). Informe de exploración detallada de Cobre en el Yacimiento Yagruma. Oficina Nacional de Recursos Minerales. La Habana.

Álvarez-Ortiz, I.; Cruz-Gámez, E.M.; Lastra-Rivero, J.F.; Velasco-Tapia, F. (2019). Alteraciones relacionadas con la mineralización sulfurosa de los yacimientos Júcaro y Yagruma, occidente de Cuba. Minería y Geología, 35(3), 270-288.

Amorós, J.L.; Lunar, R.; Tavira, P. (1981). Jarosite: a silver bearing mineral of the gossan of the Rio Tinto (Huelva) and La Union (Cartagena, Spain). Mineralium Deposita, 16(2), 205-213. https://doi. org/10.1007/BF00202735

Andreu, E.; Torró, L.; Proenza, J.A.; Domenech, C.; García-Casco, A.; Villanova de Benavent; C.; Chávez, C.; Espaillat, J.; Lewis, J.F. (2015). Weathering profile of the Cerro de Maimón VMS deposit (Dominican Republic): textures, mineralogy, gossan evolution and mobility of gold and silver. Ore Geology Reviews, 65(Part 1), 165-179. https://doi.org/10.1016/j. oregeorev.2014.09.015

Barrios, E. (1995). Presentación de las características de la región Bahía Honda. Pinar del Río. Cuba. Oficina Nacional de Recursos Minerales. La Habana.

Brodoboi, A.; Rusanov, V.; Lubennicova, T.; Lobanov, S. (1987). Informe resultados del levantamiento aerogeofísico complejo en el territorio de la provincia de Pinar del Río (Sector Pinar del Río Sur y Este). Oficina Nacional de Recursos Minerales. La Habana.

Cáceres, D. (1997). Estructura geológica y pronóstico preliminar para metales básicos más Barita en la parte central de las Alturas Pizarrosas del Sur, Pinar del Río. Ph.D. Tesis. Universidad de Pinar del Río, Cuba.

Cazañas-Díaz, X.; Torres-Zafra, J.L.; Lavaut-Copa, W.; Cobiella-Reguera, J.L.; Capote-Marrero, C.R.; González-Acosta, V.; López-Kramer, K.; BravoPatterson, F.; Llanes, A.I.; González-Castellanos, D.; Ríos-Araujo, Y.; Ortega-Rodríguez, Y.; Yasmany-Torres, R.; Pantaleón-Vento, G.; Torres- 
La Rosa, M.; Figueroa-Guanche, D. (2017). Memoria explicativa del mapa metalogénico de la República de Cuba a escala 1: 250 000. Instituto de Geología y Paleontología. Servicio Geológico de Cuba. Ministerio de Energía y Minas. Cuba.

Cobiella-Reguera, J.L. (2008). Reconstrucción palinspástica del paleomargen mesozoico de América del Norte en Cuba occidental y el sudeste del Golfo de México. Implicaciones para la evolución del SE del Golfo de México. Revista Mexicana de Ciencias Geológica, 25(3), 382-401.

Cruz-Gámez, E.M.; Simón-Méndez, A. (1997). Principales rasgos del complejo de basaltos en la región de Bahía Honda, Pinar del Río. Minería y Geología, 14(3), 51-57.

Díaz-Martínez, R. (2010). La pequeña minería del oro en Cuba: historia, metalogenia y perspectiva. Boletín de la Sociedad Geológica Mexicana, 62(1), 187-198. https://doi.org/10.18268/ BSGM2010v62n1a11

Emsbo, P.; Seal, R.R.; Breit, G.N.; Diehl, S.F.; Shah, A.K. (2016). Sedimentary exhalative (sedex) zinclead-silver deposit model. U.S. Geological Survey. Scientific Investigations Report 2010-5070-N, 57 p. https://doi.org/10.3133/sir20105070N

Escobar, E. (1973). Completamiento del cálculo de reservas y exploración orientativa del yacimiento Júcaro. Oficina Nacional de Recursos Minerales. La Habana.

Estévez-Cruz, E. (2000). 3D Orebody modelling and resource estimation of the Júcaro deposit, Pinar del Río, Cuba. Minería y Geología, 17(2), 27-29.

Fernández de Lara, R.; Cáceres-Govea, D.; Martín, R.; Denis, R.; Blanes, J. (2001). Influencia de la tectónica regional en la formación de yacimientos metálicos en la Provincia de Pinar del Río. IV Congreso Cubano de Geología y Minería, La Habana, Cuba.

Freyssinet, P.; Itard, Y. (1997). Geochemical mass balance of gold under various tropical weathering conditions: application to exploration. Exploration 97: Fourth Decennial International Conference on Mineral Exploration.
Instituto de Geología y Paleontología. (2013). Léxico Estratigráfico de Cuba. Servicio Geológico de Cuba. Ministerio de Energía y Minas. Cuba. 459p.

Iturralde-Vinent, M.A. (2012). Compendio de Geología de Cuba y del Caribe. Segunda Edición. Editorial CITMATEL.

Kalinin, Y.A.; Palyanova, G.A.; Bortnikov, N.S.; Naumov, E.A.; Kovalev, K.R. (2018). Aggregation and differentiation of gold and silver during the formation of the gold-bearing weathering crusts (on the example of Kazakhstan deposits). Doklady Earth Sciences, 482(1), 1193-1198. https://doi. org/10.1134/S1028334X18090131

Kim, B.J.; Cho, K.H.; Lee, S.G.; Park, C.Y.; Choi, N.C.; Lee, S. (2018). Effective gold recovery from nearsurface oxide zone using reductive microwave roasting and magnetic separation. Metals, 8(11). https://doi.org/10.3390/met8110957

Kulikov, A.A. (1971). Informe sobre los resultados de los trabajos de búsqueda y exploración de menas cupríferas realizados en el yacimiento Júcaro y zonas adyacentes en 1968-1971 en la parte oriental de la provincia de Pinar del Río. Oficina Nacional de Recursos Minerales. La Habana.

Lepin, O.V.; Ariosa, J.D. (1986). Cálculo de reservas de minerales útiles. En: Búsqueda, Exploración y Evaluación Geólogo-Económica de Yacimientos Minerales Sólidos. Segunda Parte. Editorial Pueblo y Educación.

Martínez, D.; Vázquez, M.; Chang, J.C.; Denis, R.; Fernández, O.; Fernández de Lara, R.; Barrios, E.; Peláez, R. (1991). Informe sobre los resultados del levantamiento geológico y prospección a escala 1: 50000 Pinar - Habana. Oficina Nacional de Recursos Minerales. La Habana.

Maximov, A. (1978). Trabajos acompañantes en la región Bahía Honda. Oficina Nacional de Recursos Minerales. La Habana.

Mormil, G.; Norman, A. (1980). Mapa geológico a escala 1: 250000 de la A. C. C. y el Informe TTP Pronóstico de Cobre Júcaro - Buena Vista (1992). Oficina Nacional de Recursos Minerales. La Habana. 
Mormil, G.; Norman, A.; Varvarov, A.; Shosiriev, V.; Linares, E. (1980). Geología y metalogenia de la provincia de Pinar del Río. Tomo II, Ciudad de la Habana. Oficina Nacional de Recursos Minerales. La Habana.

Nelson, C.E.; Proenza, J.A.; Lewis, J.F.; LópezKramer, J. (2011). The metallogenic evolution of the Greater Antilles. Geológica Acta, 9(3-4), 229264. https://doi.org/10.1344/105.000001741

Ozdemir, A.; Sahinoglu, A. (2018). Important of gossans in mineral exploration: A case study in Northern Turkey. International Journal of Earth Science and Geophysics, 4(1). http://doi. org/10.35840/2631-5033/1819

Pardo, G. (1975). Geology of Cuba. In: A.E.M. Nairn; F.G. Stehli (eds.). The Gulf of Mexico and the Caribbean (pp. 553-615). Springer. https://doi. org/10.1007/978-1-4684-8535-6_13

Pérez-Vázquez, R.G. (2000). Pronóstico de metales preciosos (Ay y Ag) en el Campo Mineral Matahambre. Tesis de Maestría. Universidad de Pinar del Río, Cuba.

Pérez-Vázquez, R.G.; Estévez-Cruz, E.; RomeroEspinosa, M.S. (2017). Modelación descriptiva del yacimiento Santa Lucía (Zn-Pb-Ba), Pinar del Río (Cuba). Boletín de Geología, 39(2), 83-93. https://doi.org/10.18273/revbol.v39n2-2017006

Proenza, J.A.; Melgarejo, J.C. (1998). Una introducción a la metalogenia de Cuba bajo la perspectiva de la tectónica de placas. Acta Geológica Hispánica, 33(1-4), 89-131.

Pszczólkowski, A. (1981). El banco carbonatado jurásico de la Sierra de los Órganos, Provincia de Pinar del Río; su desarrollo y situación paleotectónica. Ciencias de la Tierra y el Espacio 3, 37-46.

Pushcharovsky, Y.M. (1988). Mapa geológico de la República de Cuba a escala 1:250 000. Academias de Ciencias de Cuba y la USSR. En: M.A. Iturralde-Vinent (ed.). 2012. Compendio de Geología de Cuba y del Caribe. Segunda Edición. DVD-ROM. Editorial CITMATEL, La Habana, Cuba.
Simón, A. (1987). Zonación metalogénica del territorio de Cuba Occidental. III Encuentro Científico Técnico de Geología. La Habana, Cuba.

Soloviev, O.N.; Skidan, S.A.; Pankratov, A.P.; Skidan, I.K. (1964). Comentarios sobre el mapa magnetométrico de Cuba. Departamento Científico de Geología. Instituto Cubano de Recursos Minerales.

Taylor, G.F.; Sylvester, G.C. (1982). Analysis of a weathered profile on sulfide mineralization at Mugga Mugga, western Australia. Journal of Geochemical Exploration, 16(2), 105-134. https:// doi.org/10.1016/0375-6742(82)90023-1

U.S. Geological Survey, (2020). Mineral commodity summaries 2020. U.S. Geological Survey. https:// doi.org/10.3133/mcs2020

Vila-Sánchez, A.R.; Díaz-Martínez, R.; Proenza, J.A.; Melgarejo, J.C. (2004). Caracterización morfológica, textural y composicional de las partículas de oro reveladas en placeres marinos de las playas Mejías y Jiguaní (NE de Cuba Oriental). Geociências, 23(1-2), 43-53.

Vologdin, R.H.; Stepanov, V.M. (1967). Informe sobre los resultados de los trabajos de búsqueda para el cobre realizado en la parte oriental de la provincia de Pinar del Río 1965 - 1966. Oficina Nacional de Recursos Minerales. La Habana.

Yesares, L.; Aiglsperger, T.; Sáez, R.; Almodóvar, G.R.; Nieto, J.M.; Proenza, J.A.; Gómez, C.; Escobar, J.M. (2015). Gold Behavior in supergene profiles under changing redox conditions: The example of the Las Cruces deposit, Iberian Pyrite Belt. Economic Geology, 110(8), 2109-2126. https://doi.org/10.2113/econgeo.110.8.2109

Zhang, Y. (2020). Preliminary study on the characteristics of Ductile Shear Zone Type Gold Deposit. IOP Conference Series: Earth and Environmental Science, 440.

Fecha de recibido: 06 de mayo de 2020

Fecha de aprobado: 23 de octubre de 2020 